San Jose State University

SJSU ScholarWorks

Master's Theses

Master's Theses and Graduate Research

1994

\title{
Applications of new variational principle to calculate electronic states for lower dimensional semiconductor quantum wells
}

Donald Lee Tripp

San Jose State University

Follow this and additional works at: https://scholarworks.sjsu.edu/etd_theses

\section{Recommended Citation}

Tripp, Donald Lee, "Applications of new variational principle to calculate electronic states for lower dimensional semiconductor quantum wells" (1994). Master's Theses. 877.

DOI: https://doi.org/10.31979/etd.h9wc-m8d2

https://scholarworks.sjsu.edu/etd_theses/877

This Thesis is brought to you for free and open access by the Master's Theses and Graduate Research at SJSU ScholarWorks. It has been accepted for inclusion in Master's Theses by an authorized administrator of SJSU ScholarWorks. For more information, please contact scholarworks@sjsu.edu. 


\section{INFORMATION TO USERS}

This manuscript has been reproduced from the microfilm master. UMI films the text directly from the original or copy submitted. Thus, some thesis and dissertation copies are in typewriter face, while others may be from any type of computer printer.

The quality of this reproduction is dependent upon the quality of the copy submitted. Broken or indistinct print, colored or poor quality illustrations and photographs, print bleedthrough, substandard margins, and improper alignment can aơversely affect reproduction.

In the unlikely. event that the author did not send UMI a complete manuscript and there are missing pages, these will be noted. Also, if unauthorized copyright material had to be removed, a note will indicate the deletion.

Oversize materials (e.g., maps, drawings, charts) are reproduced by sectioning the original, beginning at the upper left-hand corner and continuing from left to right in equal sections with small overlaps. Each original is also photographed in one exposure and is included in reduced form at the back of the book.

Photographs included in the original manuscript have been reproduced xerographically in this copy. Higher quality $6^{\prime \prime} \times 9^{\prime \prime}$ black and white photographic prints are available for any photographs or illustrations appearing in this copy for an additional charge. Contact UMI directly to order.

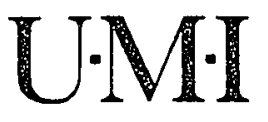

University Microfilms International

A Bell \& Howell Information Company 300 North Zeeb Road. Ann Arbor, MI 48106-1346 USA

$313 / 761-4700 \quad 800 / 521-0600$ 
Order Number 1959060

Applications of new variational principle to calculate electronic states for lower dimensional semiconductor quantum wells

Tripp, Donald Lee, M.S.

San Jose State University, 1994

Copyright (C1994 by Tripp, Donald Lee. All rights reserved.

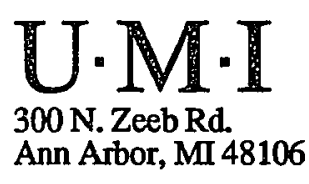


.. 


\title{
APPLICATIONS OF NEW VARIATIONAL PRINCIPLE TO CALCULATE ELECTRONIC STATES FOR LOWER DIMENSIONAL SEMICONDUCTOR QUANTUM WELLS
}

\author{
A Thesis \\ Presented to \\ The Faculty of the Department of Physics \\ San Jose State University \\ In Partial Fulfillment \\ of the Requirements for the Degree \\ Master of Science
}

by

Donald Lee Tripp

August, 1994 
(C) 1994

Donald Lee Tripp ALL RIGHTS RESERVED 
APPROVED FOR THE DEPARTMENT OF PHYSICS

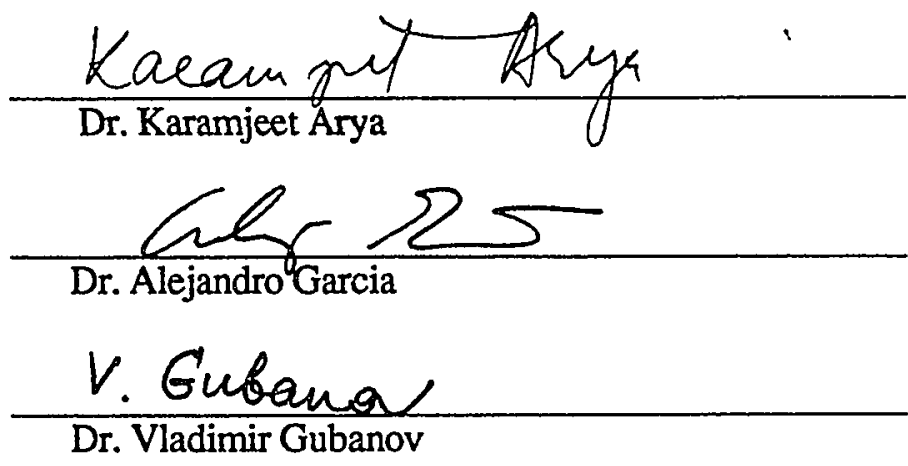

APPROVED FOR THE UNIVERSITY

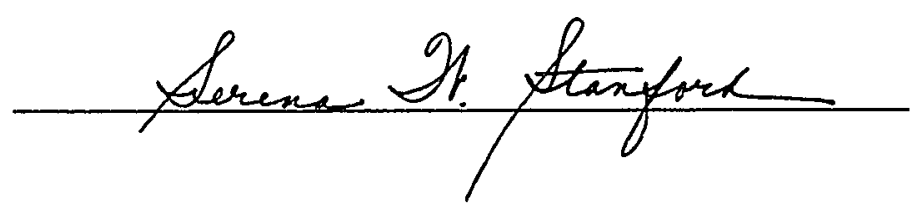




\begin{abstract}
APPLICATIONS OF NEW VARIATIONAL PRINCIPLE TO CALCULATE ELECTRONIC STATES FOR LOWER DIMENSIONAL SEMICONDUCTOR QUANTUM WELLS
\end{abstract}

\author{
by
}

\author{
Donald L. Tripp
}

\begin{abstract}
Quantum effects associated with lower dimensional semiconductor quantum wells have been observed and the focus of much attention in recent years. As a result of quantum confinement, these structures exhibit increased binding energies.
\end{abstract}

In this thesis, we study the electronic states of confined quantum systems using a new variational principle where one is free to choose any arbitrary trial wavefunction. The trial function need not satisfy any particular boundary conditions.

The binding energy of the ground state of various quantum well structures is calculated as a function of the size of the quantum well. Numerical results are discussed and compared with results obtained by other methods. We have obtained results in good agreement with other methods. Based on our studies, we find that the new variational principle is a valuable technique to calculate the electronic states for confined quantum systems. 
To my mother and father 


\section{Acknowledgements}

I would like to gratefully acknowledge the support and guidance of Dr. K. Arya, and to acknowledge the helpul discussions with Dr. A. Garcia and Dr. V. Gubanov. 


\section{TABLE OF CONTENTS}

\section{CHAPTER 1 Introduction}

1.1 Interest in Semiconductor Quantum Wells .......................................... 1

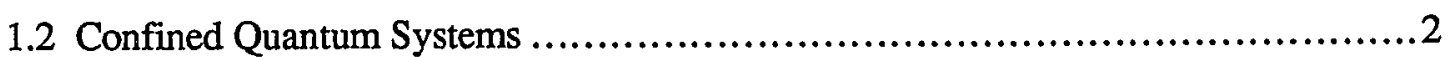

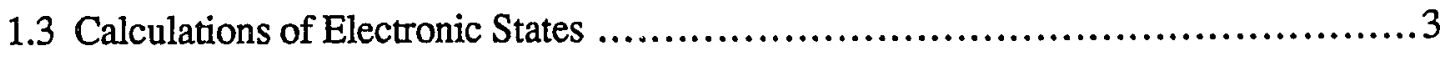

1.4 Importance of a New Variational Principle ............................................4

\section{CHAPTER 2 Semiconductor Quantum Wells}

2.1 Lower Dimensional Quantum Wells ...............................................6

2.1.1 Electronic States of the 2D Semiconductor Single Quantum Well ............ 8

2.1.2 Electronic States of 1D Quantum Wires and 0D Quantum Boxes ............11

2.2 Coulombic Bound States in Semiconductor Quantum Wells ...........................13

\section{CHAPTER 3 New Variational Principle}

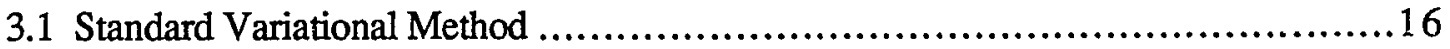

3.2 Derivation of the New Variational Principle .........................................18

3.3 Determination of Electronic States ...................................................21

\section{CHAPTER 4 Applications of the New Variational Method}

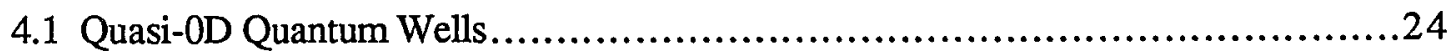

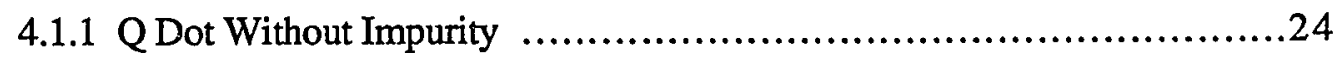

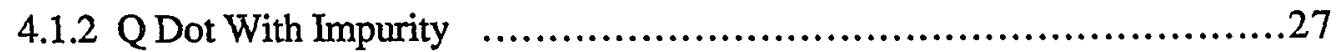

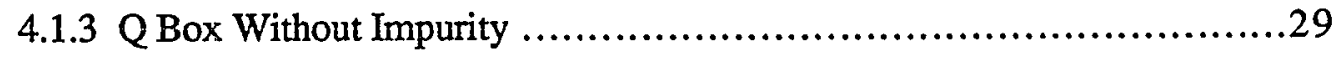

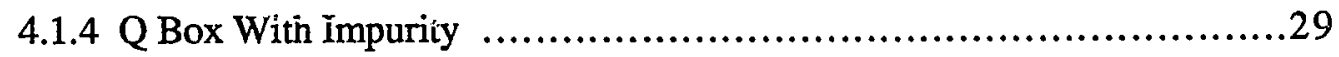

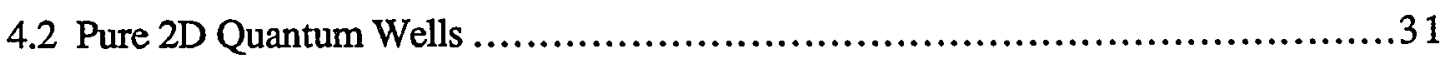

4.2.1 QW Disc Without Impurity ..................................................... 31

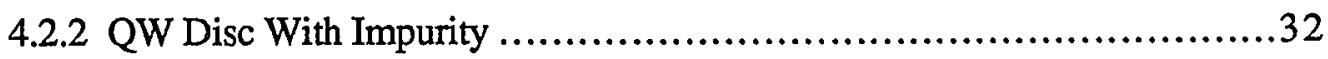

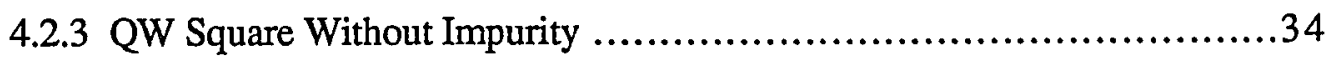

4.2.4 QW Square With Impurity ................................................34

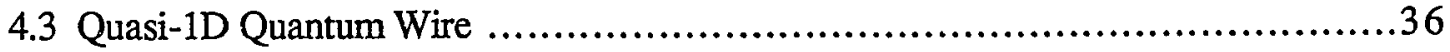

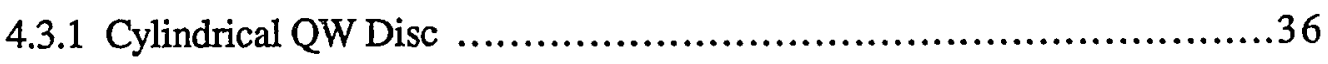

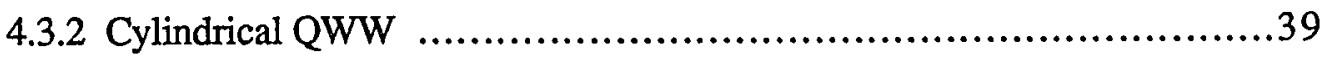




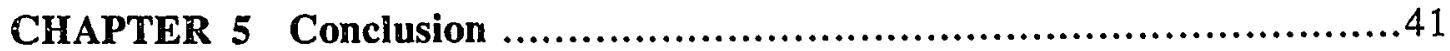

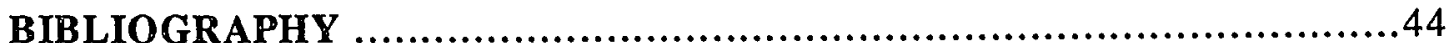

\section{APPENDIX}

C program for OD Q Dot Energy Calculation . .47 


\section{CHAPTER 1:}

\section{Introduction}

The studies of carrier confinement in quantum wells in the early 1970s have led to important developments ir semiconductor physics and device technology. Recently, semiconductor physics research is active in the area of lower dimensional confined quantum systems, ${ }^{1-6}$ partly because of advances in the ability to make ultrathin semiconductor devices (Nanotechnology). The Nanotechnology of semiconductor devices has revealed some surprising electrical and optical properties such as an increase of the electron mobility and an increase in the exciton binding energy. These properties are produced by quantum confinement effects not previously observed in semiconductor devices.

Consequently, much attention has been focused in the direction of semiconductor quantum wells, 3,7-12 quantum well wires,, $313-19$ and quantum well dots, ${ }^{20-23}$ which are the building blocks of the newest semiconductor devices such as the semiconductor quantum well laser and the high electron mobility transistor (HEMT). 3,24 The fabrication of better devices ${ }^{25-28}$ will require an imprioved understanding of semiconductor quantum wells.

\subsection{Interest in Semiconductor Quantum Wells}

In bulk or three-dimensional (3D) semiconductors, the electron mobility is severely limited by the presence of impurities. For example, when an electric field is applied to a semiconductor, the conductance of electrons is hindered by the scattering of electrons off of impurities. However, to make semiconductor devices impurities must be added.

On the other hand, if the amount of impurities can be increased and at the same time the amount of scattering decreased then faster, more efficient electrical devices can be made. A remarkable property of the quantum well $(\mathrm{QW})$ semiconductor is that the impurities can be isolated from the carriers. Hence, electron mobility is greatly enhanced. This is especially important for the modulation doped QW. 
In addition, photoluminescence studies have shown the existence of increased excitonic peaks, broader and redshifted, in semiconductor QW absorption spectrum. This is indicative of an increase in the exciton binding energy. The stability of excitons is an essential part of quantum well wire (QWW) and quantum dot (Q Dot) lasers.

Furthermore, the efficiency of semiconductor QW lasers is much better than conventional semiconductor lasers as shown below.

- Conventional laser efficiency $\quad \approx 20 \%$

- Quantum laser efficiency

- QW laser

- QWW laser

- Q Dot laser $\approx 40-50 \%$

$\approx 60-70 \%$

$>90 \%$

QW lasers are now available commercially and QWW lasers are being made in the lab. However, Q Dot lasers have not yet been fabricated, though stimulated emission has been observed recently. It is thought that to make a laser chip, an efficiency of greater than $90 \%$ is needed, otherwise the heat generated would destroy the chip. A Q Dot laser meets this criteria. Consequently, much effort is currently directed at understanding the mechanisms involved in and affecting Q Dot laser efficiency.

One of the primary reasons for such high efficiencies in quantum well devices is the experimentally observed dependence of binding energy on semiconductor dimensions. As the dimensions of the semiconductor decreases, the impurity (or exciton) binding energy increases. This is known as the quantum size effect. ${ }^{29}$ Thus, it is important to understand the physics of confined systems in order to build smaller devices.

\subsection{Confined Quantum Systems}

It is possible to grow layers of different semiconductors coherently if their lattice constants are nearly equal. These are called semiconductor heterostructures and can be viewed as a single crystal structure. ${ }^{30}$ Each semiconductor has a different band gap, and at the semiconductor-semiconductor interface (heterojunction) this difference in the band gaps produces a potential well. 
Thus, in the presence of the potential well, the electrons (holes) are confined in a two-dimensional (2D) semiconductor QW. If the dimensions of the heterostructure are further reduced, e.g., by cutting a heterostructural slab into thin strips, the electrons (holes) are confined in a one-dimensional (1D) semiconductor quantum well known as a quantum well wire (QWW). Further reducing the dimensions of the heterostructure, e.g., subdividing the heterostuctural strips into small cubes, results in the confinement of electrons (holes) in a zero-dimensional (OD) quantum well known as a quantum box ( $Q$ Box) or equivalently a $Q$ Dot.

Advances in techniques such as Molecular Beam Epitaxy, Liquid Phase Epitaxy, Chemical Vapor Deposition, and especially Electron Beam Nanolithography have led to the fabrication of submicron semiconductor structures. These techniques can produce layers of semiconductor material as thin as $2-100$ nanometers. ${ }^{24}$

In these lower dimensional systems vihere the sample size approaches the deBroglie wavelength of the electron, quantum mechanical effects, i.e., quantum interference, quantum scattering, and the quantum size effect, become important because the electron wavefunction is strongly affected by the sample boundary. ${ }^{24}$ It is important, therefore, to be able to solve the Schrodinger equation for confined quantum systems.

\subsection{Calculations of Electronic States}

One of the main difficulties in the studies of confined quantum systems is the calculation of electronic states. The technique most commonly used to calculate energy levels is the standard variational method. Binding energy calculations using the standard variational method have been done, for example, in the case of the QW, 8,12 QWW,13-15 and Q Dot. ${ }^{22}$ Usually calculations of the binding energy cannot, except in some simple cases, be solved exactly. One must, therefore, resort to approximation methods and the calculations can be very difficult.

The calculations of binding energies in lower dimensional quantum systems are important in light of the potential applications in devices for optical computers and optical communication. The calculations for the ground state binding energy of a simple lower dimensional quantum system, using the conventional variational method, were done as 
early as 1955 by Kohn and Luttinger for the case of a pure two-dimensional Hydrogen atom - yielding a binding energy four times that of the three dimensional case. ${ }^{31}$

\subsection{Importance of a New Variational Principle}

More recent attempts to solve the effective-mass Schrodinger equation, using the standard variational method8,12-15,22 for the semiconductor QW, QWW, and Q Dot have been hindered by the requirement of using wavefunctions that match the boundary conditions for the system in question. Usually, the resulting Schrodinger equation is not separable and exact solutions cannot be found.

However, a new variational principle, introduced by K. R. Brownstein in 1993,1 allows the use of trial wave functions - that do not obey the boundary conditions - to calculate the energy eigenvalues of confined quantum systems. This method eliminates the constraint of matching boundary conditions and is promising as a powerful technique in finding the energy eigenvalues of confined quantum systems. The future application of this principle may prove fruitful. 25-28 However, being a new method, it has not as yet been tested except for two simple cases: a confined Hydrogen atom, and a particle confined to a quarter circle.

In an attempt to better understand the limits and applicability of Brownstein's variational principle, we will apply this variational method to a number of simple lower dimensional quantum systems. The following systems are considered:

- ODQDot

- ODQBox

- Pure 2D QW Disc

- Pure 2D QW Square

- 1D Cylindrical QW Disc

- 1D Cylindrical QWW

Using this new variational principle to calculate the electronic states of confined quantum systems, and in particular the ground state energy, we shall find that the new variational method produces results in good agreement with experimental measurements, ${ }^{28}$ 
and theoretical results obtained from other methods. $2,5,13,15,21$ In the following chapters we expand on the ideas discussed above.

In Chapter 2, we will explain physically the concept of a 2D QW, derive the eigenfunctions and eigenvalues (electronic states) for a $2 \mathrm{D}$ semiconductor single $\mathrm{QW}$ and compare the 2D QW energy levels with bulk semiconductor energy levels. Similarly, we will also give the energy levels for $1 \mathrm{D}$ and $\mathrm{OD}$ quantum systems, concentrating on the ground state confinement energy of these systems. Finally, we will discuss how the coulombic interaction affects the energy levels in the semiconductor QW.

In Chapter 3, we first give a brief review of the stardard variational principle and then discuss and derive the new variational principle which is applicable to confined quantum systems. Following this derivation, we discuss the procedure to use this principle to solve the corresponding secular equation and obtain the energy solutions.

In Chapter 4, we calculate the electronic states of various confined quantum systems. We concentrate on the ground state impurity levels for these systems. Graphs of impurity binding energy versus quantum well thickness describe the behavior of the quantum size effect.

Chapter 5 summarizes the results and includes further discussion on the merits and disadvantages of the new variational principle. Also, we give a brief look at possible future applications of this principle. 


\section{CHAPTER 2: $\quad$ Semiconductor Quantum Wells}

Heterostructures contain single or multiple heterojunctions and are usually grown epitaxially to create alternating layers of two different semiconductor materials. The difference in the band gaps at each heterojunction interface creates a potential barrier at the interface. For multiple heterojunctions, multi-quantum-well superlattices are produced.5,32 We will consider the case of a double heterojunction which results in a single quantum well structure.

The simplest and most often used single quantum well heterostructure is the GaAs QW. This is made by cladding a layer of GaAs in between two layers of GaAlAs as depicted in Figure 2-1(a). The band gap of the GaAlAs layers is greater than or equal to the $\mathrm{GaAs}$ band gap and is largely controlled by changing the concentration level of $\mathrm{Al} .{ }^{32}$ This amount of concentration is indicated by a subscript $x$. Thus, the higher the concentration of $\mathrm{Al}$ in the GaAlAs layers, the greater the band gap difference at the heterojunctions. If an electron (or hole) is present in the $\mathrm{GaAs}$ layer then it will experience a square well potential as shown in Figure 2-1(b). In the following sections, we will discuss the single QW heterostructure and other quantum well structures, considering only the electron unless otherwise stated.

\subsection{Lower Dimensional Quantum Wells}

In terms of a quantum model, semiconductor heterostructures are relatively simple. A structure where the confinement of the electron is limited in the $\mathrm{x}, \mathrm{y}$, and $\mathrm{z}$ directions can be described in terms of a three dimensional square well model. This type of structure is called a quasi-0D QW, Q Box, or Q Dot, respectively. In a structure where the electron is free in one direction and confined in the other two directions, a two dimensional square well model can be used to describe the motion of the electron. This kind of structure is called a quasi-1D QW or QWW.

The simplest type of structure consists of an electron confined in one direction only. Figure 2-1(a) illustrates an electron confinement in one direction provided the GaAs layer is 
(a)

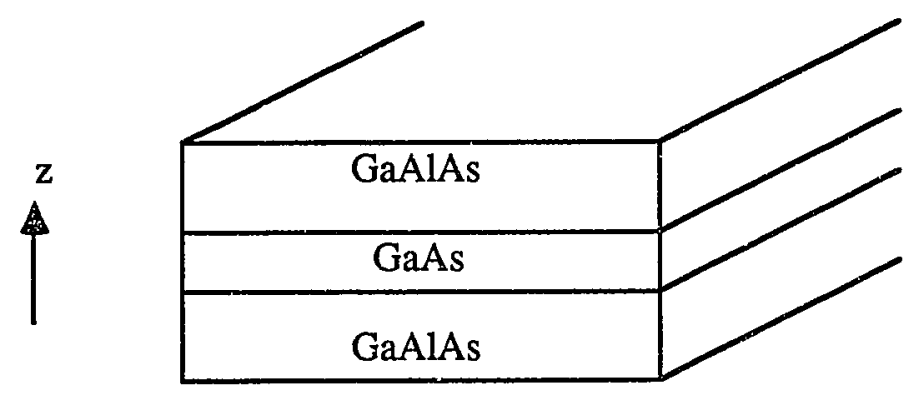

(b)

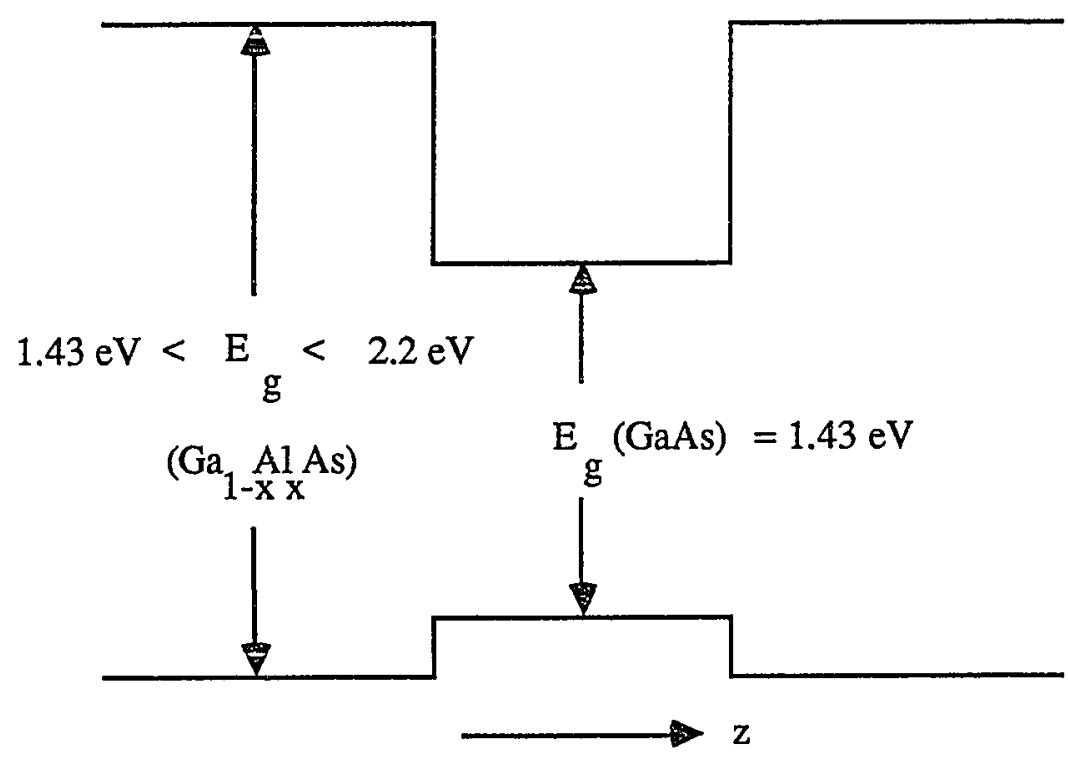

Figure 2-1: (a) GaAs heterostructure, (b) GaAs QW. 
thin (on the order of $200 \AA$ or less) and that it is sandwiched between two semi-infinite layers of $\mathrm{Ga}_{1-\mathrm{x}} \mathrm{Al}_{\mathrm{X}} \mathrm{As}$. A one dimensional square well model can then be used to describe the quantum mechanics of the electron. The structure is known as a quasi-2D QW or simply a QW.

\subsubsection{Electronic States of the 2D Semiconductor Single Quantum Well}

We consider a 2D GaAs-Ga $-\mathrm{x} \mathrm{Al}_{\mathrm{X}} \mathrm{As} \mathrm{QW}$ heterostructure with the growth axis in the $\mathrm{z}$ direction, and the $\mathrm{GaAs}-\mathrm{Ga}_{1-\mathrm{x}} \mathrm{Al}_{\mathrm{X}} \mathrm{As}$ heterojunction interfaces perpendicular to the $\mathrm{x}$ and $y$ directions. Let the GaAs layer thickness be $\mathrm{L}$. An electron (hole) in the conduction (valence) band of the GaAs layer is free to move in the $\mathrm{x}$ and $\mathrm{y}$ directions, but restricted in the $\mathrm{z}$ direction. The electron motion in the GaAs layer can be obtained by solving the single particle Schrodinger equation in a square well potential approximation. A sketch of confined potential in the case of GaAs single QW is shown in Figure 2-2.

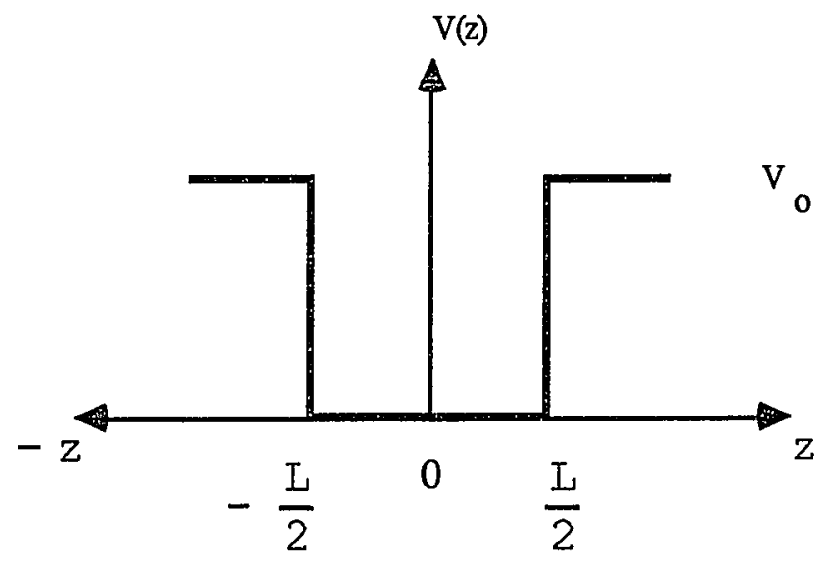

Figure 2-2: GaAs single QW along conduction band edge.

In the following, we will use the effective mass approximation and consider the motion of a conduction electron with effective mass $m_{c}$. The Schrodinger equation for the electron is

$$
\left[-\frac{\hbar^{2}}{2 m_{c}} \nabla^{2}+V(z)\right] \Psi(r)=E \Psi(r)
$$


where

$$
\begin{array}{ll}
V(z)=0 & \text { for }-L / 2 \leq z \leq L / 2 \\
V(z)=V_{0} & \text { otherwise }
\end{array}
$$

Equation (2-1) can be solved by separating it into $x-, y-$, and z-parts. The electron is still free in the $\mathrm{x}$ and $\mathrm{y}$ directions so one can write

$$
\Psi(r)=e^{i x_{x} x^{i k} e^{y}} z(z)
$$

Using Eq. (2-2) in Eq. (2-1) we obtain

$$
-\frac{\hbar^{2}}{2 m_{c}} \frac{\partial^{2}}{\partial z^{2}} Z(z)+V(z) Z(z)=E_{z} Z(z)
$$

where

$$
E_{z}=E-\frac{\hbar^{2} k^{2}}{2 m_{c}}
$$

Equation (2-3) can be solved for both cases of infinite and finite potential $\mathrm{V}_{0} .{ }^{33}$ However, we here give results for the simple case of infinite potential. In this case, the function $Z(z)$ goes to zero at the boundary $z= \pm L / 2$. Using this boundary condition, the normalized eigenfunctions of Eq. (2-2) and energy eigenvalues of Eq. (2-3) can be written as

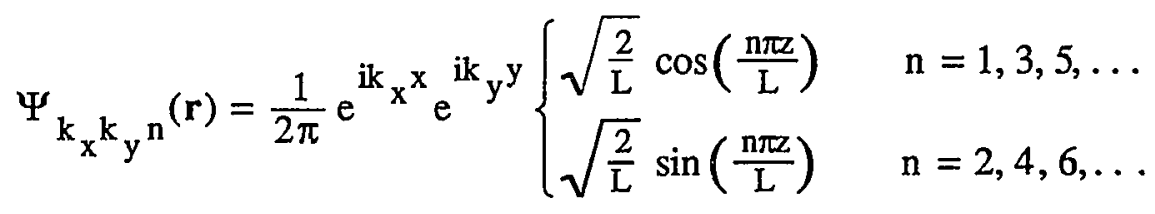

$$
\begin{aligned}
& E_{k_{x} k_{y} n}=\frac{\hbar^{2}}{2 m_{c}}\left(k_{x}^{2}+k_{y}^{2}+\left(\frac{n \pi}{L}\right)^{2}\right) \quad n=1,2,3, \ldots
\end{aligned}
$$


We observe that the ground state energy of an electron in the infinite quantum well from Eq. (2-6) is

$$
E_{001}=\frac{\hbar^{2}}{2 m_{c}}\left(\frac{\pi}{L}\right)^{2}
$$

which is finite in contrast with the free electron. For example, given a GaAs layer thickness $L$ of approximately $100 \AA$ and the electron effective mass $m_{\mathcal{C}}=0.067$, then the zero point energy $\mathrm{E}_{001}$ is approximately $5.5 \mathrm{mev}$. The energy levels for a GaAs QW are sketched in Figure 2-3.

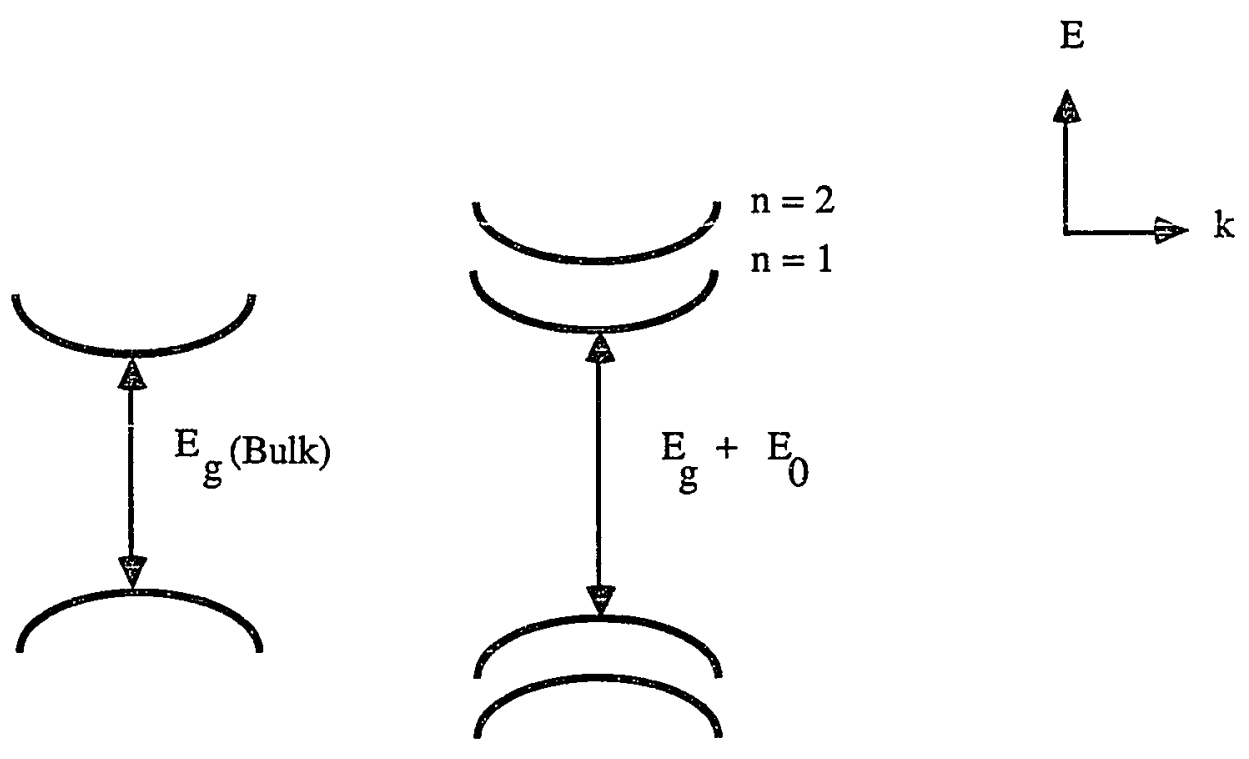

Figure 2-3: GaAs QW energy levels.

It should be pointed out that the energy band gap for the GaAs QW has increased as compared to the bulk band gap by

$$
\mathrm{E}_{0}=\frac{\hbar^{2}}{2}\left(\frac{1}{\mathrm{~m}_{\mathrm{c}}}+\frac{1}{\mathrm{~m}_{\mathrm{v}}}\right)\left(\frac{\pi}{\mathrm{L}}\right)^{2}
$$


where $\mathrm{m}_{\mathrm{c}}$ and $\mathrm{m}_{\mathrm{v}}$ are the effective masses of the conduction and valence bands, respectively. The increase in the energy band gap is due to electron confinement. Such an increase has been observed in photoluminescence and absorption experiments, by the appearance of higher energy peaks.

\subsubsection{Electronic States of 1D Quantum Wires and 0D Quantum Boxes}

The calculation of electronic states for 1D and OD QW structures are similar to those carried out in Section 2.1.1. However, for the sake of brevity, we will only discuss the energy eigenvalues.

In a semiconductor rectangular QWW the electron can move freely in the $\mathrm{x}$ direction, but is confined in the $y$ and $z$ directions. This corresponds to a small strip of GaAs with cross section dimensions $L_{y}$ and $L_{z}$. The energy eigenvalues in the infinite square well potential approximation are

$$
E_{k_{x} m n}=\frac{\hbar^{2}}{2 m_{c}}\left[k_{x}^{2}+\left(\frac{m \pi}{L_{y}}\right)^{2}+\left(\frac{n \pi}{L_{z}}\right)^{2}\right]
$$

with $m$ and $n$ being positive integers except zero The ground state energy for $k_{x}=0$, with $\mathrm{m}=\mathrm{n}=1$ and $\mathrm{L}_{\mathrm{y}}=\mathrm{L}_{\mathrm{z}}=\mathrm{L}$, becomes

$$
\mathrm{E}_{011}=2\left[\frac{\hbar^{2}}{2 \mathrm{~m}_{\mathrm{c}}}\left(\frac{\pi}{\mathrm{L}}\right)^{2}\right]
$$

One can also solve the Schrodinger equation for a cylindrical QWW where the electron is confined to an infinite cylinder of radius $R$. The eigenfunctions are Bessel functions and the corresponding eigenenergies are given by the zeroes of the Bessel function. The ground state energy for the cylindrical QWW is

$$
E_{k_{x} m n}(\min )=E_{001}=\frac{\hbar^{2}}{2 m_{c}}\left(\frac{2.4}{R}\right)^{2}
$$


for $\mathrm{k}_{\mathrm{x}}=\mathrm{m}=0$ and $\mathrm{n}=1$. Here, the value 2.4 is the first zero $\mathrm{K}_{\mathrm{mn}}$ of the Bessel function $\mathrm{J}_{\mathrm{m}}\left(\mathrm{K}_{\mathrm{mn}} \mathrm{R}\right) .{ }^{34}$

In a semiconductor $\mathrm{Q}$ Box the electron is confined in all three directions. This corresponds to a small cube of GaAs with dimensions $L_{x}, L_{y}$, and $L_{z}$. The energy eigenvalues are expressed as

$$
E_{I m n}=\frac{\hbar^{2}}{2 m_{c}}\left[\left(\frac{1 \pi}{L_{x}}\right)^{2}+\left(\frac{m \pi}{L_{y}}\right)^{2}+\left(\frac{n \pi}{L_{z}}\right)^{2}\right]
$$

with $1, m$, and $n$ positive integers. The confinement energy for $1=m=n=1$ for a cube of length $L$ is

$$
E_{111}=3\left[\frac{\hbar^{2}}{2 m_{c}}\left(\frac{\pi}{L}\right)^{2}\right]
$$

Similar to the Q Box is the Q Dot. In a Q Dot, the electron is confined to a sphere of radius $R$. One can again solve the Schrodinger equation using spherical polar coordinates. The eigenfunctions are spherical Bessel functions and the corresponding eigenenergies $\mathrm{E}_{\mathrm{nl}}$ are given by the zeroes of the spherical Bessel function. The $\mathrm{Q}$ Dot ground state energy, which corresponds to the first zero of the spherical Bessel function $\mathrm{j}_{1}\left(\mathrm{k}_{1 \mathrm{n}} \mathrm{R}\right)$ with $\mathrm{n}=1$ and $\mathrm{l}=0$, is given by

$$
E_{10}=\frac{\hbar^{2}}{2 m_{c}}\left(\frac{\pi}{R}\right)^{2}
$$

Quantum dot research is an active field, because semiconductor Q Dots can now be easily grown. Q Dots are currently fabricated by rapidly cooling hot substrates (glass) containing semiconductor (CdS) impurities. Changes in binding energy are observed as the radius of the $Q$ Dot is varied. Thus, one can better understand the quantum confinement effect by studying the $\mathrm{Q}$ Dot. 
Table 2-1 lists the ground state confinement energies of the lower dimensional quantum wells discussed above. These basic quantum well models will be used in Chapter 4 in applications of the new variational method.

Table 2-1: Ground state confinement energy of quantum well structures.

Heterostructure Ground State Confinement Energy

OD Q Dot

$\frac{\hbar^{2}}{2 m_{c}}\left(\frac{\pi}{R}\right)^{2}$

OD Q Box

$3\left[\frac{\hbar^{2}}{2 \mathrm{~m}_{\mathrm{c}}}\left(\frac{\pi}{\mathrm{L}}\right)^{2}\right]$

1D Cylindrical QWW

$\frac{\hbar^{2}}{2 \mathrm{~m}_{\mathrm{c}}}\left(\frac{2.4}{\mathrm{R}}\right)^{2}$

1D Square QWW

$2\left[\frac{\hbar^{2}}{2 m_{c}}\left(\frac{\pi}{L}\right)^{2}\right]$

2D QW

$$
\frac{\hbar^{2}}{2 m_{c}}\left(\frac{\pi}{L}\right)^{2}
$$

\subsection{Coulombic Bound States in Semiconductor Quantum Wells}

The semiconductor quantum wells discussed above are idealized quantum wells. In practice, bulk semiconductors contain impurities, either unintentionally or on purpose. Also, in photoluminescence studies, bound electron-hole pairs (excitons) are created during the photon absorption process. Thus, in bulk semiconductors there exists the presence of Hydogen-like Coulombic potentials.

We consider the case of an impurity in a pure three dimensional bulk semiconductor. Similar considerations also apply for excitons. The Hamiltonian for the electron bound to the impurity ion is given by 5 


$$
\mathrm{H}=-\frac{\hbar^{2}}{2 \mathrm{~m}^{*}} \nabla^{2}-\frac{\mathrm{e}^{2}}{\mathrm{kr}}
$$

where $\mathrm{m}^{*}$ is the effective mass of the electron (donor impurity) or hole (acceptor impurity). In Eq. (2-13), we have ignored the center of mass motion. Also, the second term on the right hand side is the impurity potential which is screened due to the static dielectric constant $K$ of the semiconductor material. The Hamiltonian in Eq. (2-13) is essentially that of a hydrogen atom problem, except that $\mathrm{m}$ is replaced by $\mathrm{m}^{*}$ and $\mathrm{e}^{2}$ is replaced by $\mathrm{e}^{2 / \kappa}$.

By comparison with the hydrogen atom problem, the energy eigenvalues for the impurity can be written as 5,35

$$
E_{n}=-\frac{m^{*} R_{H}}{m_{e} \kappa^{2} n^{2}} \quad n=1,2,3, \ldots
$$

where $R_{H}=m_{e} e^{4} / 2 \hbar^{2}$ is the Rydberg constant for the Hydrogen atom and equals $13.6 \mathrm{eV}$. For $n=1, E_{n}$ is often called the effective or impurity Rydberg. Similarly, the effective Bohr radius aimp for the impurity is

$$
\mathrm{a}^{\mathrm{imp}}=\frac{\mathrm{m}_{\mathrm{e}}}{\mathrm{m}^{*}} \kappa \mathrm{a}_{\mathrm{o}}
$$

where $\mathrm{a}_{\mathrm{o}}=\hbar^{2} / \mathrm{m}_{\mathrm{e}} \mathrm{e}^{2}=.53 \AA$ is the $\mathrm{H}$-atom Bohr radius. For GaAs, using $\mathrm{m}^{*}=0.067$ and $\kappa=12.85$, we find the ground state binding energy is approximately $5.5 \mathrm{meV}$, and the impurity Bohr radius is about $100 \AA .5$

For confined semiconductor systems, when the confined dimensions (or equivalently the layer thicknesses) of the semiconductor heterostructure are greater than the effective Bohr radius, one can still use Eq. (2-14) to obtain an approximation for the impurity energy levels. However, when the dimensions of the semiconductor are reduced to near the Bohr radius, there is an additional confinement potential V. This confinement potential must be included in the Hamiltonian of Eq. (2-13) to obtain the QW impurity levels. 
Of particular importance is the impurity problem for a pure $2 D$ semicondictor. This is essentially equivalent to a two dimensional $\mathrm{H}$-atom problem and has already been solved. 6 The impurity levels for the pure 2D semiconductor are

$$
\mathrm{E}_{\mathrm{n}}=\frac{\mathrm{m}^{*}}{\mathrm{~m}_{\mathrm{e}} \mathrm{k}^{2}} \frac{\mathrm{R}_{\mathrm{H}}}{\left(\mathrm{n}-\frac{1}{2}\right)^{2}} \quad \mathrm{n}=1,2,3, \ldots
$$

For the ground state, $n=1$, the impurity ground state reduces to 4 times the impurity Rydberg defined by Eq. (2-14) for the bulk case.

Lower dimensional semiconductor binding energy calculations have been motivated by the fact that the binding energy is strongly increased in semiconductors. Realistically, though, quasi-1D and quasi-2D systems cannot be solved analytically. Most calculations have been carried out using the standard variational method, but the calculational difficulties encountered have prevented rapid progress in theory and application. On the other hand, the new variational principle, discussed in the next chapter, is less stringent than the standard variational principle and may prove easier to use for the calculation of electronic states. 


\section{CHAPTER 3: New Variational Principle}

The Schrodinger equation can be solved exactly to obtain energy eigenvalues and eigenfunctions only for a few limited cases. Most of the time, one uses approximation methods, such as perturbation theory, or the variational method to obtain solutions of the Schrodinger equation for realistic systems. The perturbation technique is used when the Hamiltonian for the system can be split into an unperturbed $\left(\mathrm{H}_{0}\right)$ part, for which solutions of the Schrodinger equation is known, and a small perturbed $\left(\mathrm{H}^{\prime}\right)$ part.

However, when the perturbed part is not small, one usually uses the Raleigh-Ritz variational method, commonly called the standard variational method. For example, for the ground state energy calculation of the Helium atom when the repulsive Coulomb interaction between electrons is not small, the standard variational method gives much better results compared to the perturbation method. ${ }^{30}$

\subsection{Standard Variational Method}

The standard variational method is based on the following theorem..$^{33}$

Theorem: If $E_{0}$ and $\Psi_{0}(r)$ are the exact ground state energy and the ground state wavefunction, respectively, of the Schrodinger equation

$$
H \Psi(\mathbf{r})=E \Psi(\mathbf{r})
$$

and let $\Phi(\mathbf{r})$ be an arbitrary (normalized) trial function, then

$$
\mathrm{E} \geq \mathrm{E}_{\mathrm{o}}
$$

where

$$
\mathrm{E}=\langle\Phi|\mathrm{H}| \Phi\rangle
$$


Proof: We expand the trial function $\Phi(\mathbf{r})$ in terms of the complete set of exact eigenfunctions $\Psi_{\mathbf{n}}(\mathbf{r})$ :

$$
\Phi(\mathbf{r})=\sum_{\mathbf{n}} \mathrm{a}_{\mathbf{n}} \Psi_{\mathbf{n}}(\mathbf{r})
$$

where $a_{n}$ are the expansion coefficients. Using Eq. (3-4) in Eq. (3-3), we have

$$
\mathrm{E}=\sum_{\mathbf{n}}\left|\mathrm{a}_{\mathbf{n}}\right|^{2} \mathrm{E}_{\mathrm{n}}
$$

We rewrite Eq. (3-5) as

$$
E-E_{0}=\sum_{n}\left|a_{n}\right|^{2}\left[E_{n}-E_{0}\right]
$$

where $\sum\left|a_{n}\right|^{2}=1$ has been used due to normalization. Since $E_{n} \geq E_{0}$ for a bound system and $\operatorname{la}_{n} \mid 2$ is always positive, we have $E \geq E_{0}$.

The usefulness of this method is that if the trial wavefunction $\Phi(\mathbf{r})$ is made to depend on one or more variational parameters $\lambda_{i}$ then an upper bound for the ground state energy can be obtained by minimizing $E\left(\lambda_{i}\right)$. This is done by setting $\partial E / \partial \lambda_{i}=0$ and solving for $\lambda_{i}$. This gives the best possible value of $\lambda_{i}$, and therefore $E$, for the chosen trial function.

The main drawback of the variational method is that the upper bound for the ground state energy depends on the trial wavefunction. Thus, an inappropriate choice for a trial wavefunction will result in an inaccurate estimate of the ground state energy of the system in question. In spite of this, the variational method has been used extensively in the literature. It is also commonly used to obtain exciton binding energies and impurity levels for semiconductor confined systems. In this case, the trial function always satisfies the boundary conditions of the confined system. This restriction on the trial function sometimes makes the variational calculations very tedious. $4,8,12$ The new variational principle, discussed below, removes this restriction. It allows one to determine the energy eigenvalues without consideration of boundary conditions when choosing the trial function. 


\subsection{Derivation of the New Variational Principle}

Consider a particle of mass $m$, under the influence of a potential $V(r)$, and confined to a region of space $\mathbf{R}$ and bounding surface $\Sigma$ as shown below.

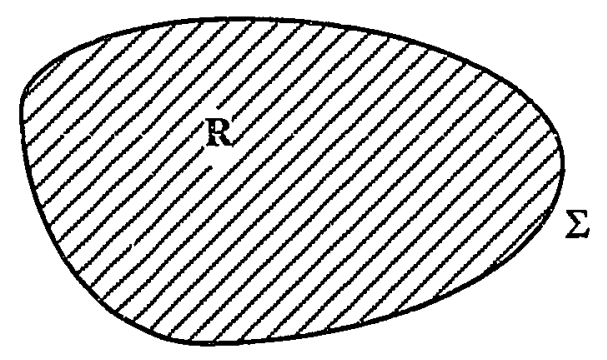

The particie is then subject to the Hamiltonian

$$
\mathrm{H}=-\frac{\hbar^{2}}{2 \mathrm{~m}} \nabla^{2}+\mathrm{V}(\mathbf{r})
$$

Let $\Psi(\mathbf{r})$ be an exact wavefunction and $E$ an exact energy eigenvalue such that

$$
\mathrm{H} \Psi(\mathbf{r})=\mathrm{E} \Psi(\mathbf{r})
$$

For all points on the surface of or outside of the region $R, \Psi(\mathbf{r})=0$. This is the boundary condition for the exact wavefunction $\Psi(\mathbb{r})$.

We choose a trial function $\Phi(\mathbf{r})$ which need not satisfy the boundary condition on or outside the bounding surface of the the region $\mathbf{R}$, that is, $\Phi(\mathbf{r})$ need not be zero on or outside the region $\mathbf{R}$. We write

$$
\Phi(\mathbf{r})=\Psi(\mathbf{r})+f(\mathbf{r})
$$

where $f(\mathbf{r})$ is the correction term to the exact function $\Psi(\mathbf{r})$. We now calculate $\langle\Phi|\mathrm{H}-\mathrm{E}| \Phi\rangle$ using Eqs. (3-7) - (3-9). In the following, the $r$ dependence of $\Psi, \Phi, f$, and $V$ will be suppressed, unless otherwise stated. We obtain 


$$
\int_{R^{-}} \Phi(\mathrm{H}-\mathrm{E}) \Phi \mathrm{d} \tau=\int_{\mathrm{R}^{-}} \Phi\left(-\frac{\hbar^{2}}{2 \mathrm{~m}} \nabla^{2} \mathrm{f}\right) \mathrm{d} \tau+\int_{\mathrm{R}^{-}} \Phi(\mathrm{V}-\mathrm{E}) \mathrm{fd} \tau
$$

where $\mathrm{d} \tau$ is the integration volume element. Also, we have used Eqs. (3-8) and (3-9) to rewrite the right hand side of Eq. (3-10). We use Green's theorem ${ }^{33}$

$$
\int_{R^{-}}\left(\Phi \nabla^{2} \hat{f}-f \nabla^{2} \Phi\right) d \tau=\int_{\Sigma}(\Phi \nabla f-f \nabla \Phi) \cdot d S
$$

to rewrite the first integral on the right hand side of Eq. (3-10). In Eq. (3-11) $\Sigma$ denotes the surface of the region $\mathbf{R}$. Now, using Eq. (3-8) to again rewrite the right hand side of Eq. (3-10), we obtain

$$
\int_{\mathrm{R}^{-}} \Phi(\mathrm{H}-\mathrm{E}) \Phi \mathrm{d} \tau=\frac{\hbar^{2}}{2 \mathrm{~m}} \int_{\Sigma}(\mathrm{f} \nabla \Phi-\Phi \nabla f) \cdot \mathrm{d} S+\int_{\mathrm{R}^{-}} \mathrm{f}(\mathrm{H}-\mathrm{E}) \mathrm{f} \mathrm{d} \tau
$$

From Eq. (3-9) we find that on the surface $\Sigma, \Phi(r)=f(r)$ because $\Psi(r)=0$ on the surface due to boundary conditions. However, in general, $\nabla \Psi$ need not be equal to $\nabla f$ on the bounding surface. Using the fact that $\Psi(r)$ vanishes on the surface we can then rewrite Eq. (3-12) as

$$
\int_{R^{-}} \Phi(H-E) \Phi d \tau=\frac{\hbar^{2}}{2 m} \int_{\Sigma}(\Phi \nabla \Phi-f \nabla f) \cdot d S+\int_{R^{-}} f(H-E) f d \tau
$$

We again use Green's theorem in the form of Eq. (3-11), to change the surface integral in Eq. (3-13) back into a volume integral. Equation (3-13) can then be expressed as 


$$
\begin{aligned}
\int_{R^{-}} \Phi(H-E) \Phi d \tau= & \frac{\hbar^{2}}{2 m} \int_{R^{-}}\left(\Phi \nabla^{2} \Phi+\nabla \Phi \cdot \nabla \Phi-f \nabla^{2} f-\nabla f \cdot \nabla f\right) d \tau \\
& +\int_{R^{-}} f(H-E) f d \tau
\end{aligned}
$$

Finally, using Eq. (3-7) for $\mathrm{H}$ and bringing all $\Phi$ dependent terms on the right hand side and $\mathrm{f}$ dependent terms to the left hand side, Eq. (3-14) becomes

$$
\varepsilon-E=\frac{\int_{R^{-}}\left[-\frac{\hbar^{2}}{2 m}\left(2 f \nabla^{2} f+\nabla f \cdot \nabla f\right)+f(V-E) f\right] d \tau}{\int_{R^{-}} \Phi^{2} d \tau}
$$

where $\varepsilon$ is defined by

$$
\varepsilon=\frac{\int_{\mathrm{R}^{-}}\left[-\frac{\hbar^{2}}{2 \mathrm{~m}}\left(2 \Phi \nabla^{2} \Phi+\nabla \Phi \cdot \nabla \Phi\right)+\Phi V \Phi\right] \mathrm{d} \tau}{\int_{\mathrm{R}^{-}} \Phi^{2} \mathrm{~d} \tau}
$$

Now, let $f \rightarrow \lambda f$ in Eq. (3-9), The right hand side of Eq. (3-9) is then quadratic in $\lambda$, and to the first order in $\lambda, \partial \varepsilon / \partial \lambda=0$. Thus, $\varepsilon$ is the stationary value of $E$, denoted by [E]. Equation (3-16) is Brownstein's variational principle for the energy $E$. If $\Phi$ vanishes 
on the surface of the region $\mathbf{R}$, then Eq. (3-16) reduces to the standard Raleigh-Ritz variational principle. However, if $\Phi$ does not vanish on the boundary surface, then the kinetic energy term is modified as in Eq. (3-16).

The advantage of the new variational principle is the freedom to choose a trial wave function. The trial function does not need to satisfy any particular boundary conditions. The disadvantage of the new variational principle is that it is not a definite minimum principle - in contrast to the standard Rayleigh-Ritz variational method. Therefore, in using the new variational method, increasing the number of variational parameters does not necessarily guarantee improved results.

\subsection{Determination of Electronic States}

To find the eigenvalues, we write the trial wavefunction $\Phi$ as

$$
\Phi(r)=\sum_{i=1}^{k} c_{i} u_{i}(r)
$$

where the $u_{i}(r)$ are $\mathrm{k}$ arbitrary real functions and the $c_{i}$ are $\mathrm{k}$ real variational parameters. We can now substitute the expression for $\Phi(\mathbf{r})$ defined by Eq. (3-17), whereby Eq. (3-16) can be written as

$$
\sum_{i, j} c_{i} c_{j}\left[\widetilde{H}_{i j}-[E] S_{i j}\right]=0
$$

where we define

$$
\begin{aligned}
& \widetilde{H}_{i j}=T_{i j}+V_{i j} \\
& T_{i j}=-\frac{\hbar^{2}}{2 m} \int_{R^{-}}\left(u_{i} \nabla^{2} u_{j}+u_{j} \nabla^{2} u_{i}+\nabla u_{i} \cdot \nabla u_{j}\right) d \tau
\end{aligned}
$$




$$
\begin{aligned}
& v_{i j}=\int_{R^{-}} u_{i} V_{j} d \tau \\
& s_{i j}=\int_{R^{-}} u_{i} u_{j} d \tau
\end{aligned}
$$

Minimizing Eq. (3-18) with respect to the variational parameters $c_{i}$, we obtain the following set of equations.

$$
\begin{aligned}
& c_{1}\left[\widetilde{\mathrm{H}}_{11}-[\mathrm{E}] \mathrm{S}_{11}\right]+\mathrm{c}_{2}\left[\widetilde{\mathrm{H}}_{12}-[\mathrm{E}] \mathrm{S}_{12}\right]+\ldots \\
& \mathrm{c}_{1}\left[\widetilde{\mathrm{H}}_{21}-[\mathrm{E}] \mathrm{S}_{21}\right]+\mathrm{c}_{2}\left[\widetilde{\mathrm{H}}_{22}-[\mathrm{E}] \mathrm{S}_{22}\right]+\ldots
\end{aligned}
$$

and so on

This set of secular equations has a solution provided

$$
\operatorname{Det}|\widetilde{H}-[E] S|=0
$$

This is a generalized eigenvalue problem. It is sometimes referred to as the algebraic eigenvalue problem ${ }^{36,37}$ and is a major problem area in numerical analysis. However, the generalized eigenvalue problem of Eq. (3-21) can be transformed into a standard eigenvalue problem provided $S$ is not singular. For example, by multiplying both sides of Eq. (3-21) by the inverse of $S$

$$
\operatorname{Det}\left|S^{-1} \widetilde{\mathrm{H}}-[\mathrm{E}] \mathrm{I}\right|=0
$$

where $I$ is the identity matrix. In general, the matrix $\mathrm{S}^{-1} \widetilde{\mathrm{H}}$ need not be symmetric. As a result, there are two possible major difficulties which might occur: (1) the eigenvalues can 
be complex, (2) the eigenvalues can be very sensitive to small changes in the matrix elements. ${ }^{36,37}$ However, these difficulties can be dealt with as described in the next chapter. We will use this approach of finding solutions to the unsymmetric eigenvalue problem given by Eq. (3-22) in the next chapter to calculate the electronic states for confined quantum systems. 


\section{CHAPTER 4: Applications of the New Variational Method}

In the previous chapter, we derived the new variational principle applicable to confined quantum systems. We will here use this method to the various cases of confined quantum systems discussed in Chapter 2 to find their ground state energies.

The eigenvalues for each case were found using a program similar to the $C$ program given in the Appendix which calculates the eigenvalues for the Q Dot. All programs were run on a $386 \mathrm{PC}$ computer. Numerically, the eigenvalues are obtained from the matrices $S$ and $\widetilde{\mathrm{H}}$ by first multiplying $S$ inverse by $\widetilde{\mathrm{H}}$ to obtain $\mathrm{S}^{-1} \tilde{\mathrm{H}}$ thereby reducing the problem to a standard eigenvalue problem. In general, $\mathrm{S}^{-1} \tilde{\mathrm{H}}$ will not be a symmetric matrix. So, to minimize rounding errors, $S^{-1} \tilde{\mathrm{H}}$ is balanced. Next, $\mathrm{S}^{-1} \tilde{\mathrm{H}}$ is reduced to Hessenberg form and then input to a $Q R$ algorithmically based routine. ${ }^{36-39}$ This routine decomposes the matrix $S^{-1} \widetilde{H}$ into the form $Q \bullet R$, where $Q$ is an orthogonal matrix and $R$ is an upper triangular matrix, and returns the eigenvalues of the original matrix $S^{-1} \tilde{\mathrm{H}}$.

\subsection{Quasi-0D Quantum Wells}

Confinement of an electron in all three dimensions corresponds to the OD quantum structures. The calculations of eigenvalues were carried out for two quantum well types: a spherical quantum well (Q Dot) and a rectangular quantum well (Q Box). Eigenvalues are obtained for a confined electron with and without the presence of an impurity.

\subsubsection{Q Dot Without Impurity}

We consider an electron confined to a sphere of radius $R$ and choose a trial wavefunction of the form

$$
\Phi(\mathbf{r})=\sum_{i=1}^{k} c_{i} u_{i}(r)
$$


with $u_{i}=r^{i-1} e^{-\lambda I}$ where $r$ is in units of $R$ and $\lambda$ is an additional dimensionless parameter used to control the decay of the wavefunction at the edge of the QDot.

To find the eigenvalues of the secular determinant of Eq. (3-21) only the matrix elements $S_{i j}$ and $T_{i j}$ need to be calculated, since in the absence of an impurity $V_{i j}=0$ so that $\widetilde{\mathrm{H}}_{\mathrm{ij}}=\mathrm{T}_{\mathrm{ij}}$. Recall, from Chapter 3, that

$$
\mathrm{T}_{\mathrm{ij}}=-\frac{\hbar^{2}}{2 \mathrm{~m}} \int_{\mathrm{R}^{-}}\left(\mathrm{u}_{\mathrm{i}} \nabla^{2} \mathrm{u}_{\mathrm{j}}+\mathrm{u}_{\mathrm{j}} \nabla^{2} \mathrm{u}_{\mathrm{i}}+\nabla \mathrm{u}_{\mathrm{i}} \cdot \nabla \mathrm{u}_{\mathrm{j}}\right) \mathrm{d} \tau
$$

Using Eq. (4-1), one can write the analytical expression for $T_{i j}$ (in units of $\hbar^{2} / 2 m$ ) as

$$
\mathbf{T}_{\mathrm{ij}}=-3 \lambda^{2} \mathrm{~T}_{\mathrm{ij}}^{0}+[3(\mathrm{i}+\mathrm{j})-2] \lambda \mathrm{T}_{\mathrm{ij}}^{1}-\left[1-2(\mathrm{i}+\mathrm{j})+\mathrm{ij}+\mathrm{i}^{2}+\mathrm{j}^{2}\right] \mathrm{T}_{\mathrm{ij}}^{2}
$$

where we define

$$
\begin{aligned}
& T_{i j}^{0}=4 \pi \int u_{i} u_{j} r^{2} d r \\
& T_{i j}^{1}=4 \pi \int u_{i}\left(\frac{1}{r}\right) u_{j} r^{2} d r \\
& T_{i j}^{2}=4 \pi \int u_{i}\left(\frac{1}{r^{2}}\right) u_{j} r^{2} d r
\end{aligned}
$$

We observe that the matrix elements $\mathrm{T}_{\mathrm{ij}}=S_{\mathrm{ij}}$. So, the eigenvalues may now be found numerically. 
We choose a unit sphere of radius $R=1$ and a decay parameter $\lambda=1$ so that on the surface of the sphere the wavefunction reduces to $e^{-1}$ of its value at the center of the sphere. We consider two cases using 4 and then 5 variational parameters, which correspond to working with $4 \times 4$ and $5 \times 5$ matrices, respectively, and compare the results. In the absence of an impurity, we find the electron ground state confinement energy $\sim 1.0$ (in units of $\hbar^{2}(\pi / R)^{2} / 2 m$ ). The expected value obtained analytically (see Table 2-1), for the ground state energy is 1 . Now, the new variational method is applicable to confined systems so to further reduce the value of the wavefunction outside the sphere we let $\lambda=2$. Table 4-1 lists the eigenvalues obtained with $\lambda=1,2$ and $R=1$ for $4 \times 4$ and $5 \times 5$ matrices.

Table 4-1: Eigenvalue spectrum for $Q$ Dot (without the impurity) for 4 and 5 variational parameters for both $\lambda=1$ and $\lambda=2$. The eigenvalues labeled by a "*" correspond to our best choice for the true ground state.

\begin{tabular}{ccc}
\hline \hline \multirow{2}{*}{ Matrix size } & \multicolumn{2}{c}{ Eigenvalues } \\
& & $\lambda=2$ \\
\hline \multirow{2}{*}{$4 \times 4$} & -19.4246 & -9.5720 \\
& $1.0014^{*}$ & $1.0010^{*}$ \\
& 4.3047 & 4.0948 \\
& 11.2582 & 13.5705 \\
& & \\
& & \\
& -49.0314 & -31.2378 \\
& $1.0160^{*}$ & $1.0001^{*}$ \\
& 4.2274 & 4.0519 \\
& 10.4131 & 9.9703 \\
& 21.0169 & 26.4029 \\
\hline \hline
\end{tabular}

The new variational principle is not a definite minimum principle. Thus, for a given number of variational parameters, the lowest eigenvalue, of the eigenvalues obtained using the above procedure, need not correspond to the true ground state. It is necessary to 
choose the correct eigenvalue. Initially, we tried comparing the (normalized) wavefunction probabilities for an electron outside of the sphere. One would expect that the true ground state energy would correspond to the wavefunction whose probability outside the sphere is minimum. However, we were unable to obtain results which consistently agreed with this assumption and were thus forced to look at the properties of the eigenvalue spectrum.

There are several noticeable properties of the eigenvalues in Table 4-1. First, there are negative eigenvalues. Second, for a given $\lambda$ and matrix size the negative eigenvalues are much lower than the other eigenvalues. Third, as $\lambda$ changes or the matrix size changes, most of the eigenvalues change considerably, except one which is close to 1.0 and it changes the least. In consideration of these properties, we choose the eigenvalue(s) labelled with a "*" in Table 4-1 as the true ground state energy of the system.

\subsubsection{Q Dot With Impurity}

We use the wavefunction of Eq. (4-1). In the presence of an impurity, located at the center of the sphere, there is an additional Coulomb potential interaction. The corresponding matrix elements are

$$
V_{i j}=-2 \cdot 4 \pi \int u_{i}\left(\frac{1}{r}\right) u_{j} r^{2} d r
$$

with $V_{i j}$ in units of $e^{2} / 2 a^{i m p}$ where $a^{i m p}$ is the effective Bohr radius. The other matrices are still given by Eqs. (4-2) - (4-5), but we now have $\tilde{\mathrm{H}}_{\mathrm{ij}}=\mathrm{T}_{\mathrm{ij}}+\mathrm{V}_{\mathrm{ij}}$.

The binding energy of the hydrogenic impurity is equal to the ground state energy of the quantum dot without the impurity minus the ground state energy with the impurity. Also, in the presence of an impurity, the electron is much more likely to be located near the center of the quantum dot; thus, we choose the decay parameter $\lambda=1$. Figure 4- 1 shows results obtained for $\lambda=1$, of the binding energy as a function of $R$, with $R$ in units of $\mathrm{a}^{\mathrm{imp}}$. At $\mathrm{R}=4$, the binding energy equals 1.5823 and the ground state energy equals 0.9654. This agrees with the results of Brownstein using the new variational method and 


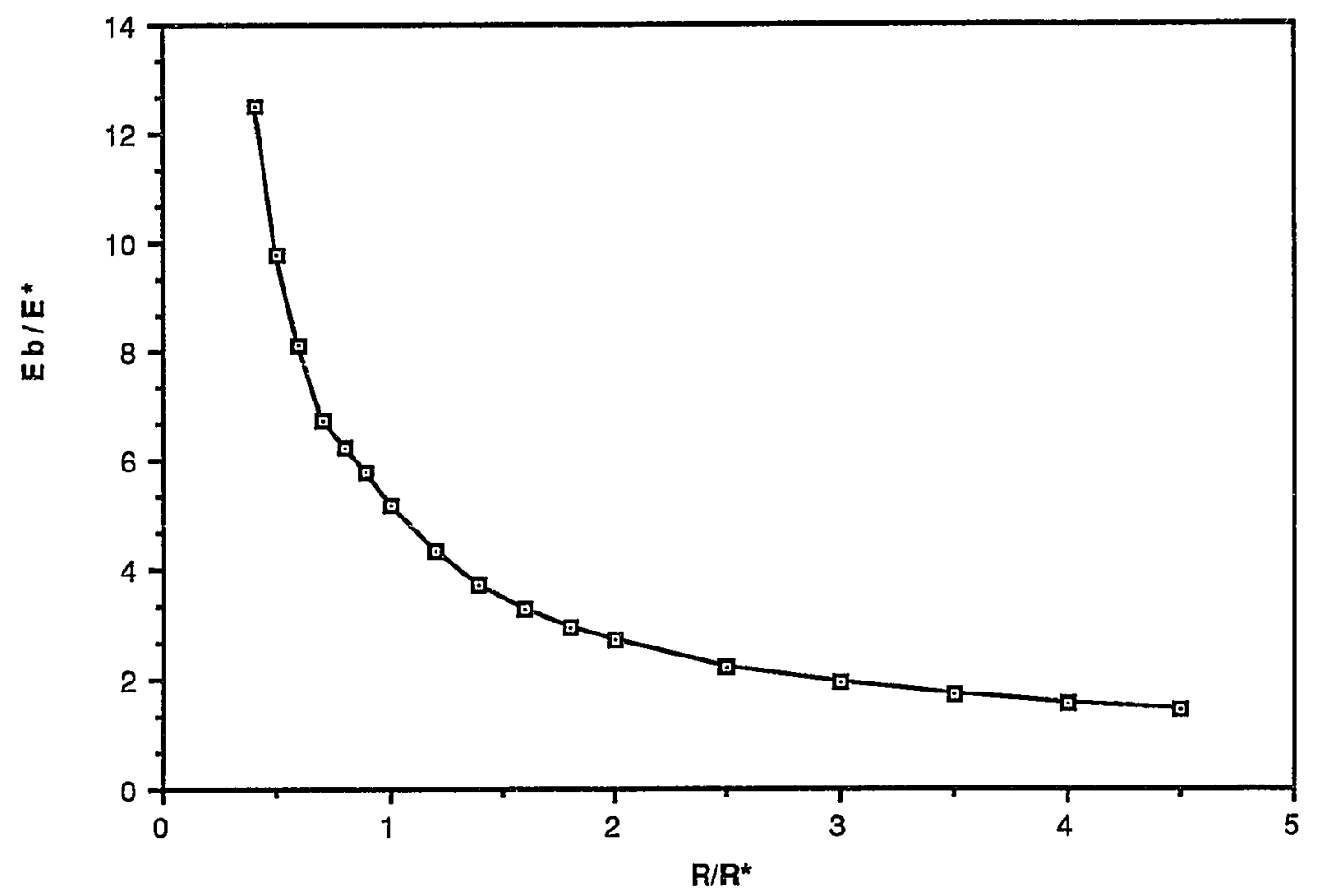

Figure 4-1: The impurity binding energy Eb of the Q Dot in units of the effective Rydberg $E^{*}$ is plotted against the dimensionless ratio of the $Q$ Dot radius $R$ to the effective Bohr radius $R^{*}$. 
with results obtained using more conventional methods. 2,21 For large $R$, the binding energy approaches 1 , as expected for an unconfined hydrogenic impurity in three dimensions.

\subsubsection{Q Box Without Impurity}

We consider an electron confined to a rectangular cube of side length $\mathrm{L}$, and origin at the center of the cube. We choose the same trial wavefunction as in Eq. (4-1), with $r=$ $\sqrt{ }\left(x^{2}+y^{2}+z^{2}\right)$ and $x, y$, and $z$ in units of $L$

The matrix elements for $T_{i j}$ and $S_{i j}$ are still given by Eqs. (4-2) - (4-5), but the integrations are now three-dimensional integrations with volume element dxdydz. A modified version of the program in the Appendix was used employing the $3 \mathrm{D}$ integration subroutine similar to quad3d() from reference [37]. We choose $\lambda=3.5$ and use three variational parameters which corresponds to working with $3 \times 3$ matrices. In the absence of an impurity, we find the electron ground state confinement energy $\sim 2.94$ (in units of $\hbar^{2}(\pi / \mathrm{L})^{2} / 2 \mathrm{~m}$ ). The analytical value (see Table $2-1$ ) for the ground state energy is 3.0 . However, as we increase the number of variational parameters, the ground state energy decreases. For example, for five variational parameters, the ground state energy decreases to 2.49 .

\subsection{4 $Q$ Box with Impurity}

In the presence of an impurity, located at the center of the cube, the potential matrix elements are

$$
V_{i j}=-2 \iint u_{i}\left(\frac{1}{r}\right) u_{j} d x d y d z
$$

where $r=\sqrt{ }\left(x^{2}+y^{2}+z^{2}\right)$ with $x, y$,and $z$ in units of the effective Bohr radius aimp. From this, the binding energy was calculated as a function of $L$ (in units of $a^{i m p}$ ). Figure 4-2 shows the results obtained for $\lambda=1$. Again for $L \gg R^{*}$, the effective Bohr radius, the 


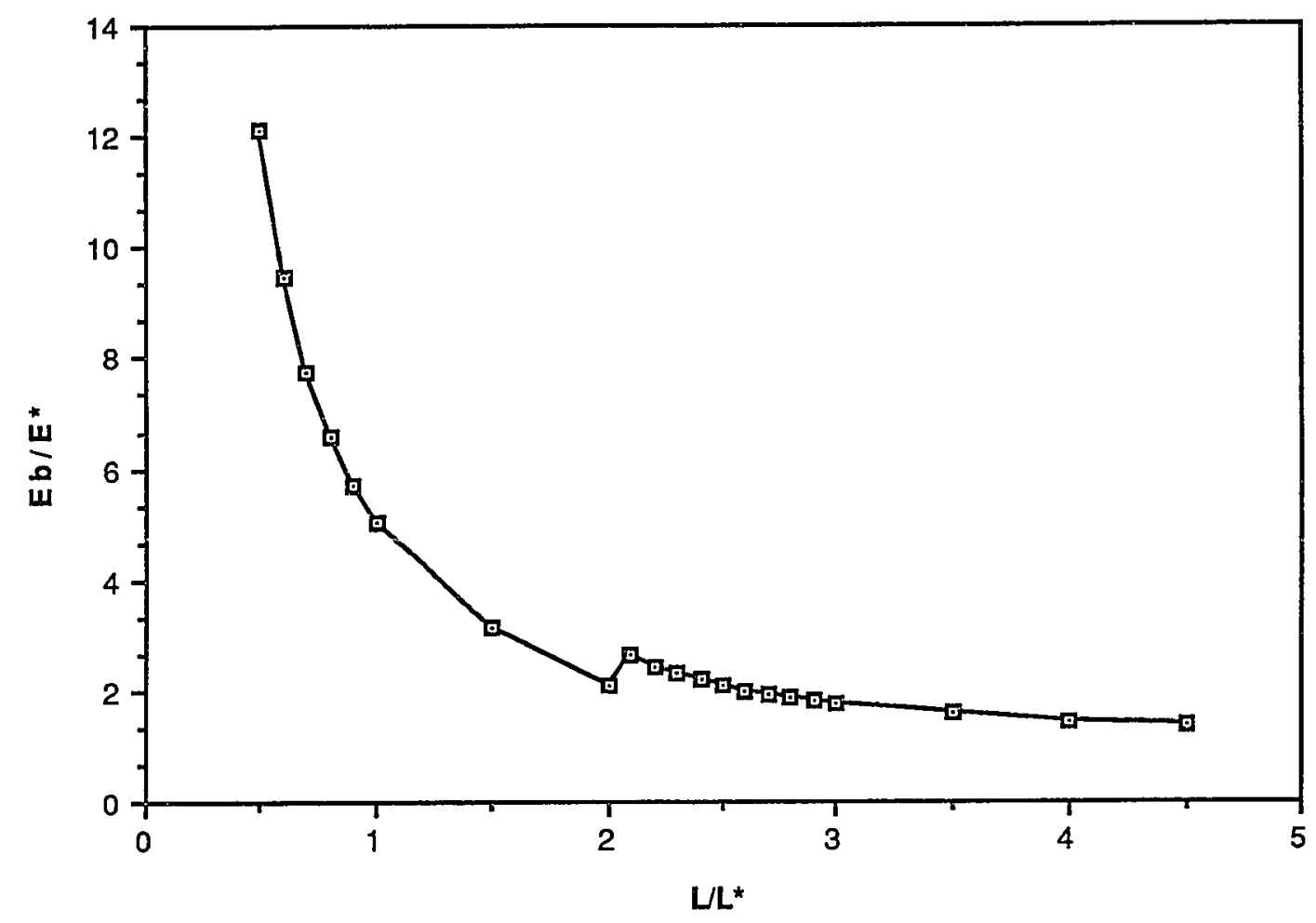

Figure 4-2: The impurity binding energy Eb of the $Q$ Box in units of the effective Rydberg $\mathrm{E}^{*}$ is plotted against the dimensionless ratio of the $\mathrm{Q}$ Box side of length $\mathrm{L}$ to two times the effective Bohr radius $L^{*}=2 R^{*}$. 
impurity binding energy approaches one effective Rydberg as for bulk semiconductors. However, it approaches $\sim 12$ for $L=R^{*}$. At $L \sim 4 R^{*}$ we observe a small bump in the binding energy. This is probably a result of performing 3D numerical integration or possibly because of choosing an inappropriate trial wavefunction.

\subsection{Pure 2D Quantum Wells}

An idealized approximation of a 1D quantum wire structure is that of an electron confined purely to two dimensions. For this model, two quantum systems are considered: a pure 2D QW Disc and a pure 2D QW Square. Both cases include an electron in the absence and presence of an impurity. As the dimensions of the QW approach infinity, an approximation to a 2D quantum well structure is achieved.

\subsubsection{QW Disc Without Impurity}

We consider a pure 2D QW Disc of radius $R$ and choose a trial wavefunction of the form

$$
\Phi(r)=\sum_{i=1}^{k} c_{i} r^{i-1} e^{-\lambda r}
$$

where $r$ is in units of $R$.

The matrix elements $T_{i j}$ (in units of $\hbar^{2} / 2 \mathrm{~m}$ ) are rewritten as

$$
T_{i j}=-3 \lambda^{2} T_{i j}^{0}+[3(i+j)-4] \lambda T_{i j}^{1}-\left[3-3(i+j)+i j+i^{2}+j^{2}\right] T_{i j}^{2}
$$

where we define

$$
\mathrm{T}_{\mathrm{ij}}^{0}=2 \pi \int \mathrm{u}_{\mathrm{i}} \mathrm{u}_{\mathrm{j}} \mathrm{rdr}
$$




$$
\begin{aligned}
& \mathrm{T}_{\mathrm{ij}}^{1}=2 \pi \int \mathrm{u}_{\mathrm{i}}\left(\frac{1}{\mathrm{r}}\right) \mathrm{u}_{\mathrm{j}} \mathrm{rdr} \\
& \mathrm{T}_{\mathrm{ij}}^{2}=2 \pi \int \mathrm{u}_{\mathrm{i}}\left(\frac{1}{\mathrm{r}^{2}}\right) \mathrm{u}_{\mathrm{j}} \mathrm{rdr}
\end{aligned}
$$

where $u_{i}=r^{i-1} e^{-\lambda r}$.

We choose a unit disc of radius $\mathrm{R}=1$, a decay parameter $\lambda=1$, and use 5 variational parameters. In the absence of an impurity, we find the electron ground state confinement energy 0.998 [in units of $\hbar^{2}(2.4 / \mathrm{R})^{2} / 2 \mathrm{~m}$ ]. This is close to the analytical value of 1 (see Table 2-1).

\subsubsection{QW Disc With Impurity}

In the presence of an impurity, located at the center of the disc, the additional Coulomb potential matrix elements are

$$
V_{i j}=-2 \cdot 2 \pi \int u_{i}\left(\frac{1}{r}\right) u_{j} r d r
$$

with $V_{i j}$ in units of $e^{2 / 2} a^{i m p}$ where $a^{i m p}$ is the effective Bohr radius. The other matrices $S_{\mathrm{ij}}$, and $\mathrm{T}_{\mathrm{ij}}$ are the same as in Eqs. (4-9) - (4-12). We plot the binding energy as a function

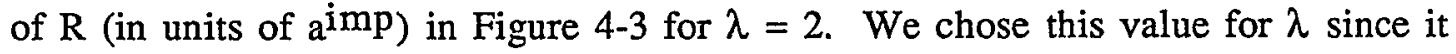
corresponds to the decay parameter of a $2 \mathrm{D}$ system and we are here interested in the limit as $R$ approaches infinity. Figure 4-3 shows that for large $R$, the binding energy approaches 4 , in agreement with the pure $2 \mathrm{D} \mathrm{H}$-atom results obtained from other methods. 6,12 


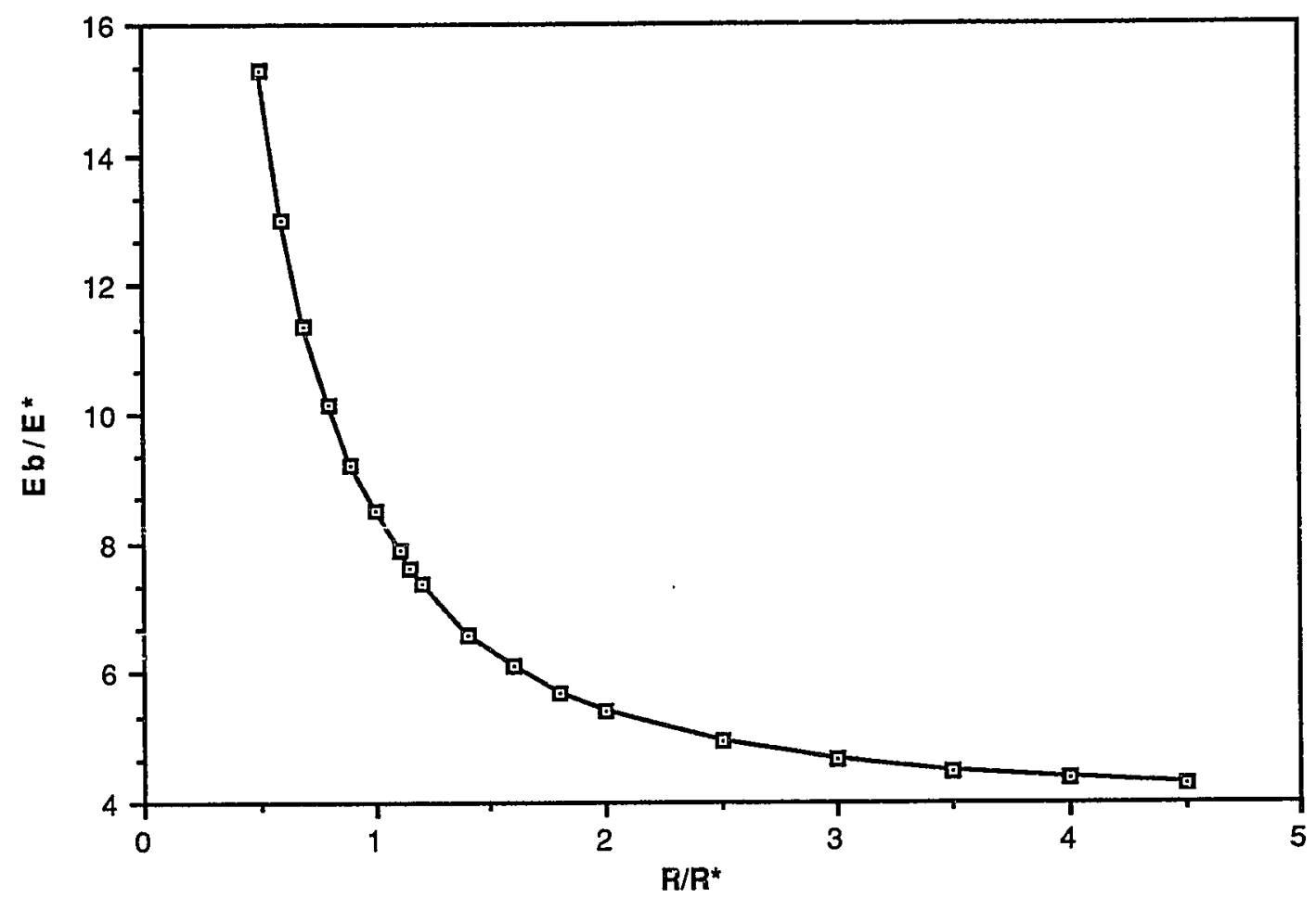

Figure 4-3: The impurity binding energy Eb of the pure 2D QW Disc in units of the effective Rydberg $E^{*}$ is plotted against the dimensionless ratio of the QW Disc radius $\mathrm{R}$ to the effective Bohr radius $R^{*}$. 


\subsubsection{QW Square Without Impurity}

We consider an electron confined to a square of side length $\mathrm{L}$, and origin at the center of the square. We choose the same trial wavefunction as in Eq. (4-7), where $r=$ $\sqrt{ }\left(x^{2}+y^{2}\right)$ and with $x$ and $y$ in units of $L$.

The matrix elements for $T_{i j}$ and $S_{i j}$ are still given by Eqs. (4-9) - (4-12), but the integrations are now two-dimensional integrations with volume element dxdy. To calculate eigenvalues, we use a modified version of the program in the Appendix employing a 2D integration subroutine analogous to the subroutine quad $3 \mathrm{~d}($ ). We choose $\lambda=3$, and use 3 variational parameters thus limiting numerical work to $3 \times 3$ matrix calculations. In the absence of an impurity, we find the electron ground state confinement energy $\sim 2.09$ (in units of $\hbar^{2}(\pi / L) 2 / 2 m$ ). The expected value (refer to Table 2-1) for the ground state energy is 2.0. As we increase the number of variational parameters, the ground state energy again decreases similar to that observed for the quantum box. For example, for five variational parameters, the ground state energy decreases to 1.80 .

\subsubsection{QW Square With Impurity}

In the presence of an impurity located at the center of the square, the $\mathrm{V}_{\mathrm{ij}}$ are given by

$$
v_{i j}=-2 \iint u_{i}\left(\frac{1}{r}\right) u_{j} d x d y
$$

where $r=\sqrt{ }\left(x^{2}+y^{2}\right)$ with $x$ and $y$ in units of the effective Bohr radius aimp. The binding energy as as a function of $L$ (in units of $\mathrm{a}^{\mathrm{imp}}$ ) for $\lambda=3$ is shown in Figure 4-4. For large $\mathrm{L}$, the binding energy levels approaches 4.0 . 


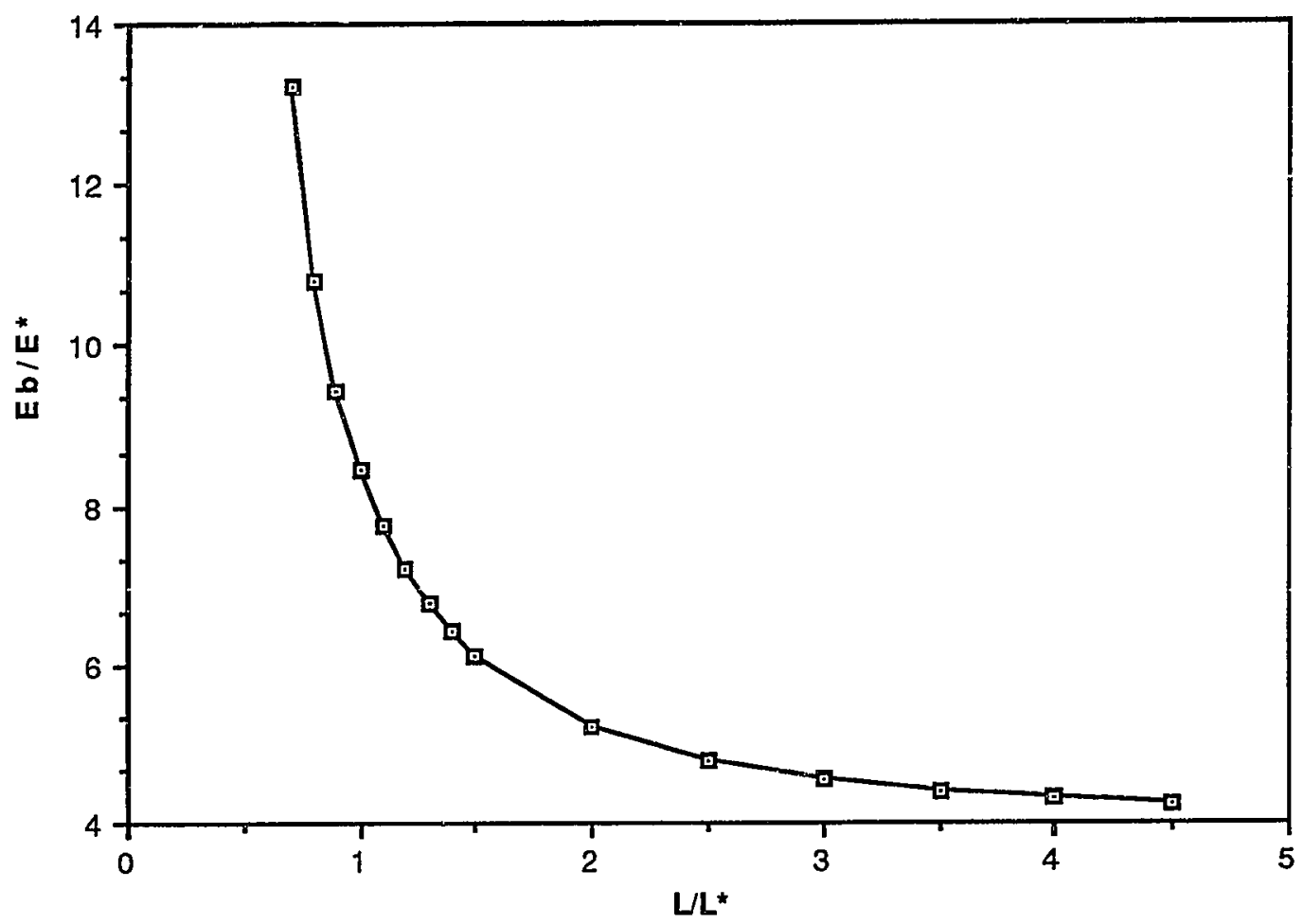

Figure 4-4: The impurity binding energy Eb of the pure 2D QW Square in units of the effective Rydberg $E^{*}$ is plotted against the dimensionless ratio of the QW Square side of length $\mathrm{L}$ to two times the effective Bohr radius $\mathrm{L}^{*}=2 \mathrm{R}^{*}$ : 


\subsection{Quasi-1D Quantum Wire}

A more realistic model of a 1D quantum wire is that of an electron confined to a cylindrical wire of radius $\rho$ and length $L$ with an impurity located at the center of the wire. We consider two limiting cases: (1) reducing the length $L$ of the wire while keeping the radius $\rho$ constant, with $\rho$ much larger than $a^{i m p}$, (2) reducing the radius $\rho$ of the wire

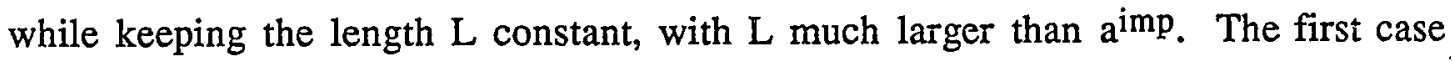
corresponds to a cylindrical QW Disc and the second to a cylindrical QWW. We first consider the case of a cylindrical QW Disc.

\subsubsection{Cylindrical QW Disc}

We choose a trial function of the form

$$
\Phi(\rho, z)=\sum_{i=1}^{k} c_{i} z^{2(i-1)} e^{-\lambda \sqrt{\rho^{2}+z^{2}}}
$$

where $\mathrm{z}$ is in the direction of the disc axis. The $\mathrm{T}_{\mathrm{ij}}$ are given by

$$
T_{i j}=-3 \lambda^{2} T_{i j}^{0}+[6(i+j)-8] \lambda T_{i j}^{1}-\left[16-14(i+j)+4\left(i^{2}+j^{2}+i j\right)\right] T_{i j}^{2}
$$

where we define

$$
\begin{aligned}
& \mathrm{T}_{\mathrm{ij}}^{0}=2 \pi \int \mathrm{u}_{\mathrm{i}} \mathrm{u}_{\mathrm{j}} \rho d \rho d z \\
& \mathrm{~T}_{\mathrm{ij}}^{1}=2 \pi \iint \mathrm{u}_{\mathrm{i}}\left(\frac{1}{\sqrt{\rho^{2}+\mathrm{z}^{2}}}\right) \mathrm{u}_{\mathrm{j}} \rho \mathrm{d} \rho \mathrm{dz}
\end{aligned}
$$




$$
\mathrm{T}_{\mathrm{ij}}^{2}=2 \pi \iint \mathrm{u}_{\mathrm{i}}\left(\frac{1}{\mathrm{z}^{2}}\right) \mathrm{u}_{\mathrm{j}} \rho \mathrm{d} \rho \mathrm{dz}
$$

where $u_{i}=z^{2(i-1)} e^{-\lambda} \sqrt{ }\left(\rho^{2}+z^{2}\right)$. In addition, the $V_{i j}$ are given by

$$
V_{i j}=-2 \cdot 2 \pi \iint u_{i}\left(\frac{1}{\sqrt{\rho^{2}+z^{2}}}\right) u_{j} \rho d \rho d z
$$

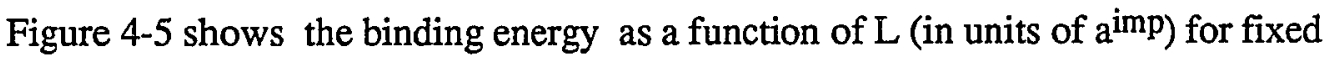
$p=10 \mathrm{a}$ imp and $\lambda=1$. For large $\mathrm{L}$, we see that the binding energy approaches the threedimensional bulk value of 1.0. As $\mathrm{L}$ is decreased the binding energy increases. The binding energy versus quantum well thickness $\mathrm{L}$ displays the same behavior obtained by Bastard $^{5}$ using the standard variational method. In the limit $\mathrm{L}$ goes to zero, which corresponds to a pure $2 \mathrm{D}$ system, the binding energy appears to approach the pure $2 \mathrm{D}$ binding energy of 4.0. 


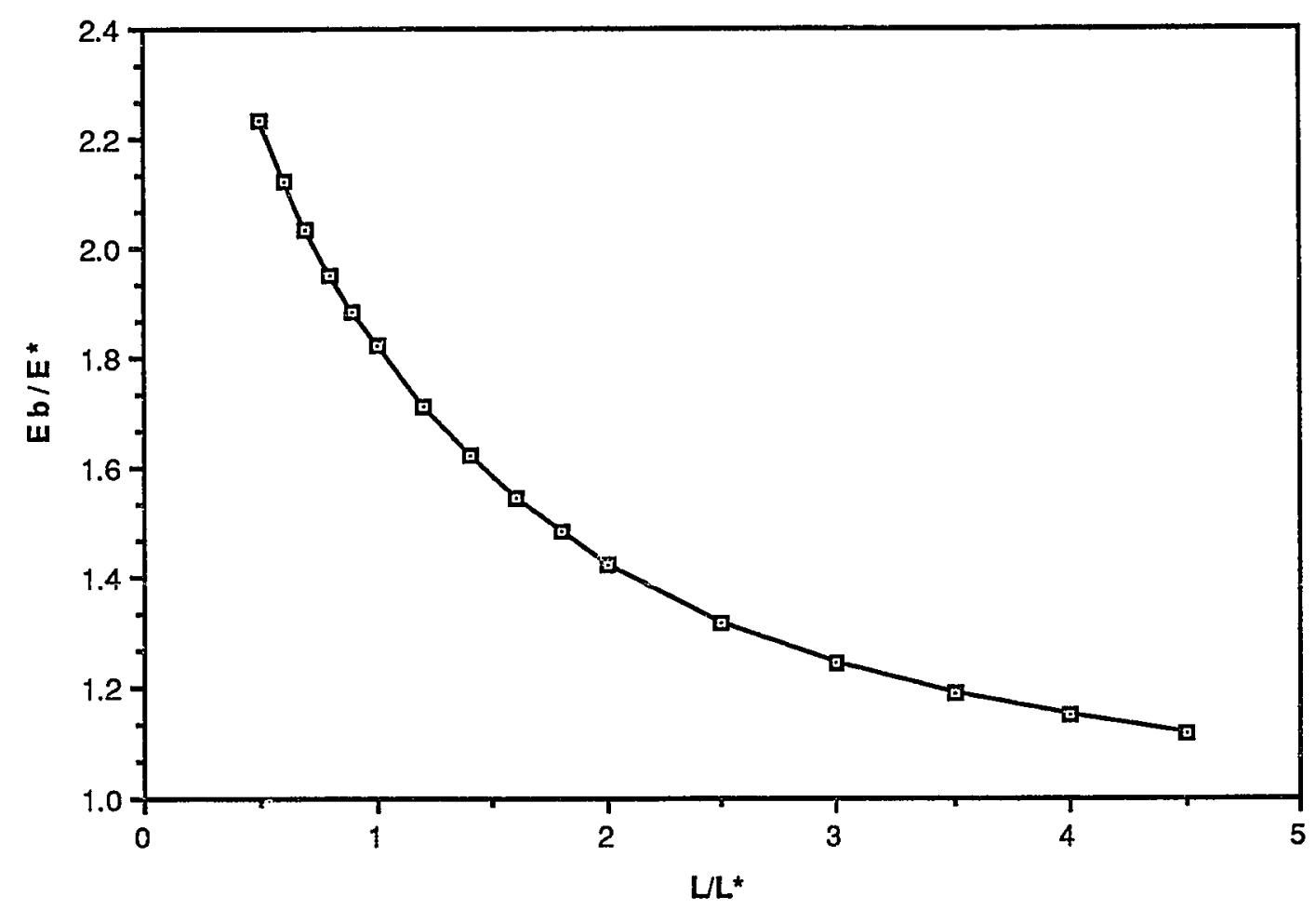

Figure 4-5: The impurity binding energy Eb of the cylindrical QW Disc in units of the effective Rydberg $E^{*}$ is plotted against the dimensionless ratio of the cylindrical $Q W$ Disc length $\mathrm{L}$ to two times the effective Bohr radius $\mathrm{L}^{*}=2 \mathrm{R}^{*}$. The radius of the Disc is kept fixed at $p=10 R^{*}$. 


\subsubsection{Cylindrical QWW}

Finally, we consider a trial wavefunction of the form

$$
\Phi(\rho, z)=\sum_{i=1}^{k} c_{i} \rho^{2(i-1)} e^{-\lambda \sqrt{\rho^{2}+z^{2}}}
$$

The $T_{i j}$ are given by

$$
\mathrm{T}_{\mathrm{ij}}=-3 \lambda^{2} \mathrm{~T}_{\mathrm{ij}}^{0}+[6(\mathrm{i}+\mathrm{j})-8] \lambda \mathrm{T}_{\mathrm{ij}}^{1}-\left[12-12(\mathrm{i}+\mathrm{j})+4\left(\mathrm{i}^{2}+\mathrm{j}^{2}+\mathrm{ij}\right)\right] \mathrm{T}_{\mathrm{ij}}^{2}
$$

where we define $\mathrm{T}^{0}{ }_{\mathrm{ij}}$ and $\mathrm{T}^{1}{ }_{\mathrm{ij}}$ similarly as in Eqs. (4-17) and (4-18) respectively, but with the $u_{i}$ defined by $u_{i}=\rho^{2(i-1)} e^{-\lambda} \sqrt{ }\left(\rho^{2}+z^{2}\right)$, and where we define the $T^{2}{ }_{i j}$ elements by

$$
\mathrm{T}_{\mathrm{ij}}^{2}=2 \pi \iint \mathrm{u}_{\mathrm{i}}\left(\frac{1}{\rho^{2}}\right) \mathrm{u}_{\mathrm{j}} \rho \mathrm{d} \rho \mathrm{dz}
$$

Similarly, the $\mathrm{V}_{\mathrm{ij}}$ are defined as in Eq. (4-20), but with the $u_{\mathrm{i}}$ defined as above.

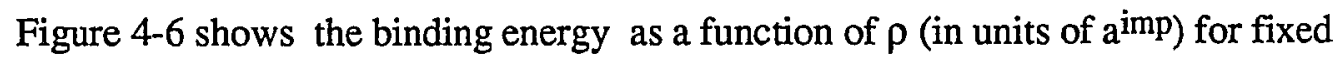
$\mathrm{L}=10 \mathrm{a} \mathrm{imp}$ and $\lambda=1$. The binding energy approaches the three-dimensional bulk value of 1.0 for large $\rho$, and as $\rho$ is decreased the binding energy increases. For example, it approaches $\sim 3$ for $\rho$ equal to one effective Rydberg. 


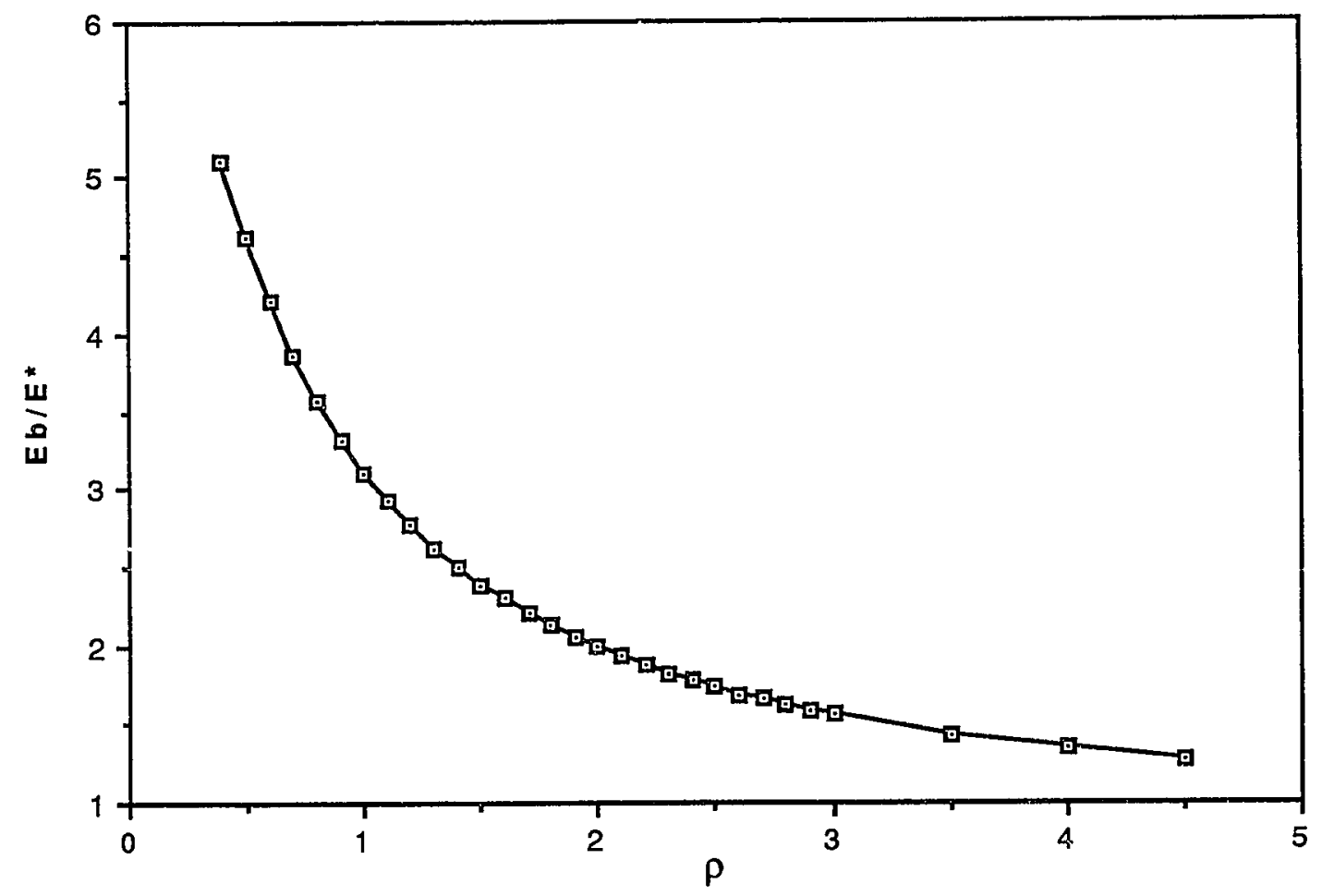

Figure 4-6: The impurity binding energy Eb of the cylindrical QWW in units of the effective Rydberg $E^{*}$ is plotted against the QWW radius $\rho$, with $\rho$ in units of the effective Bohr radius $R^{*}$. The length of the Wire is kept fixed at $L=10 R^{*}$. 


\section{CHAPTER 5:}

\section{Conclusion}

In summary, we have applied a new variational method to calculate the electronic states for lower dimensional semiconductor quantum wells. We have considered, in particular, the ground state impurity levels of the OD Q Dot and Q Box, the pure 2D QW Disc and QW Square, and two limiting cases of a 1D cylindrical QWW - a cylindrical QW Disc and an infinitely long cylindrical QWW. We shall now summarize our results and observations made, and evaluate the usefulness and validity of the new variational principle.

In our calculations, we have used the infinite square well potential and the effective mass approximations. We have found that the binding energy for an impurity located at the center of a QW structure is a function of the confinement of the carriers along the quantized direction(s). As the dimensions of the confined system decrease, the binding energy increases. The general behavior of this quantum size effect is somewhat independent of the shape of the semiconductor $\mathrm{QW}$, but depends more on the extent of confinement.

In comparing the binding energy of the $Q$ Dot to the $Q$ Box as a function of quantum confinement, we find that for comparable dimensions, the binding energies are qualitatively the same. Similarly, in comparing the binding energy of the pure 2D QW Disc to that of the pure 2D QW Square, we also find this behavior. In addition, for both the $0 \mathrm{D}$ and pure 2D systems, as the dimensions of the system become large, and the effect of confinement less, the binding energies approach their expected analytical 3D and 2D results of one effective and four effective Rydbergs, respectively.

On the other hand, as the dimensions of the OD and pure 2D structures approach zero, we find that the binding energy in the pure $2 \mathrm{D}$ quantum models is larger than the binding energy obtained for the $0 \mathrm{D}$ systems. For example, at one effective Bohr radius, the binding energy of the pure 2D QW Disc is roughly nine effective Rydbergs as compared to five effective Rydbergs for the $Q$ Dot. We suggest that as the dimensions of the pure 2D systems are reduced and likewise for the OD systems, the electron becomes 
more confined in the pure $2 \mathrm{D}$ systems. This results in the comparatively greater binding energy.

For the limiting cases of a 1D quantum wire, we find the binding energy for the quasi-2D cylindrical QW Disc is less than that of the infinitely long QWW. For large disc radius, as the size of the disc edge is reduced, one approaches a pure 2D structure and a corresponding binding energy of four effective Rydbergs; in the case of an infinitely long cylindrical QWW as the radius of the wire diminishes, the increase in binding energy exhibits the characteristic 1D behavior. The plots of binding energy as a function of quantum confinement for these systems reflect this.

Similarly, we see that the $1 \mathrm{D}$ binding energy is less than the $0 \mathrm{D}$ binding energy as the confinement in the quantized direction(s) is increased. In fact, we can compare the binding energy for the cylindrical QW Disc, cylindrical QWW, and Q Dot which correspond to $2 \mathrm{D}, 1 \mathrm{D}$, and $\mathrm{OD}$ quantum systems, respectively. For a confinement equal to one effective Bohr radius, we have obtained a binding energy of approximately two, three, and five effective Rydbergs, respectively. This is in good agreement with previously obtained results obtained using more conventional methods such as the standard variational method. $2,9,13$

In deriving the above results, we have used relatively simple trial wavefunctions and obtained reasonable results. This is the main advantage of the new variational method. Rather than using Bessel functions, spherical Bessel functions, sine and cosine functions or some other functions that satisfy the boundary conditions applicable to the system at hand, we were free to choose much simpler trial functions.

The new variational method yields good results for the cases considered above where we were able to choose the true ground state energy solutions based on physical intuition. However, the future application of this principle requires a better method of choosing the energy solutions. Since the principle is not a definite minimum principle, for more complicated applications it may not be as easy to select the true energy eigenvalue.

The increased binding energy and subsequent enhanced stability is an attractive feature of semiconductor confined quantum wells. This is of major importance for the 
potential use of high mobility electronic devices, excitonic gain induced lasers, and the further miniaturization of semiconductor devices.

Furthermore, we suggest that future studies most suited to the use of the new variational principle include the following topics: the effects of roughness on electron mobility, the effects of electric and magnetic fields, enhanced excitonic induced laser gain, ${ }^{28}$ and new quantum well structures such as the T-shaped ${ }^{26,27}$ and Crossed quantum wells. ${ }^{25}$ The new variational principle should prove useful in further developing our understanding of lower dimensional semiconductor structures. 


\section{BIBLIOGRAPHY}

1. K. R. Brownstein, "Variational Principle for Confined Quantum Systems", Phys. Rev Lett. 71, 1427 (1993).

2. C. M. Hsiao, W. N. Mei, and D .S. Chuu, "Quantum Confinement Effect on the Impurity States of a Spherical Confining System", Solid State Commun. 81, 807 (1991).

3. H. Tanksalwalla, "Semiconductor Quantum Well and Quantum Wire Lasers", (Graduate Thesis, Department of Physics: San Jose State University, 1991).

4. A. D'Andrea and R. Del Sole, "Excitons in Semiconductor Confined Systems", Solid State Commun. 74, 1121 (1990).

5. G. Bastard, "Wave Mechanics Applied to Semiconductor Heterostructures", (New York: Wiley, 1988).

6. H. I. Ralph, "The Electronic Absorption Edge in Laser Type Crystals", Solid State Commun. Vol 3, pp 303-306, (1965).

7. J. Ding, H. Jeon, T. Ishihara, M. Hagerott, and A.V. Nurmikko, "Excitonic Gain and Laser Emission in ZnSe-Based Quantum Wells", Phys. Rev. Lett. 69, 1707 (1991).

8. R. L. Greene, K. K. Bajaj, and D. E. Phelps, "Energy Levels of Wannier Excitons in GaAs-Ga1-XAlX Quantum-Well Structures", Phys. Rev. B 29, 1807 (1983).

9. G. Bastard, E. E. Mendez, L. L. Chang, and L. Esaki, "Exciton Binding Energy in Quantum Wells", Phys. Rev. B 26, 1974 (1982).

10. R. L. Greene and K. K. Bajij, "Binding Energies of Wannier Excitons in GaAs-Ga1-xAl $\mathrm{X}$ Quantum Well Structures", Solid State Commun. 45, 831 (1983).

11. R. C. Milier, D. A. Kleinman, W. T. Tsang, and A. C. Gossard, "Observation of the Excited Level of Excitons in GaAs Quantum Wells", Phys. Rev. B 24, 1134 (1981).

12. G. Bastard, "Hydrogenic Impurity States in a Quantum Well: A Simple Model", Phys. Rev. B 24, 4714 (1981).

13. P. Csavinszky and H. Oyoko, "Binding Energy of On-Axis Hydrogenic and NonHydrogenic Donors in a GaAs/Ga $1-\mathrm{Al} \mathrm{Al}_{\mathrm{x}} \mathrm{As}$ Quantum-Well Wire of Circular Cross Section", Phys. Rev. B 43, 9262 (1990). 
14. J. W. Brown and H. N. Spector, "Hydrogen Impurities in Quantum Well Wires", J. Appl. Phys. 59, 1179 (1986).

15. G. W. Bryant, "Hydrogenic Impurity States in Quantum-Well Wires", Phys. Rev. B 29, 6632 (1984).

16. P. F. Yuh and K. L. Wang, "One-dimensional transport in quantum well wire-high electron mobility transistor", Appl. Phys. Lett. 49, 1738 (1986).

17. G.W. Bryant, "Hydodenic Impurity States in Quantum-Well Wires: Shape Effects", Phys. Rev. B 31, 7812 (1985).

18. J. Lee and H. N. Spector, "Impurity-Limited Mobility of Semiconducting Thin Wire", J. Appl. Phys. 54, 3921 (1983).

19. V. K. Arora, "Quantum Size Effect in Thin-Wire Transport", Phys. Rev. B 23, $5611(1981)$.

20. Ch. Sikorski and U. Merkt, "Spectroscopy of Electronic States in InSb Quantum Dots", Phys. Rev. Lett. 62, 2164 (1989).

21. J. Gorecki and W. B. Brown, "Variational Boundary Perturbation Theory for Enclosed Quantum Systems", J. Phys. B 22, 2659 (1989).

22. G. W. Bryant, "Excitons in Quantum Boxes: Correlation Effects and Quantum Confinement", Phys. Rev. B 37, 8763 (1988).

23. M .A. Reed, J. N. Randall, R. J. Aggarwal, R. J. Matya, T. M. Moore, and A. E. Wetsel, "Observation of Discrete Electronic States in a Zero-Dimensional Semiconductor Nanostructure", Phys. Rev. Lett. 60, 535 (1987).

24. H. Heinrich, G. Bauer, F. Kuchar (Eds), "Physics and Technology of SubMicron Structures", (New York: Springer-Verlag, 1988).

25. R. L. Schult, D. G. Ravenhall, and H. W. Wyld, "Quantum Bound States in a Classically Unbound System of Crossed Wires", Phys Rev B 39, 5476 (1988).

26. A. R. Goni, L. N. Pfeiffer, K. W. West, A Pinczuk, H. U. Baranger, and H. L. Stormer, "Observation of Quantum Wire Formation at Intersecting Quantum Wells", Appl Phys. Lett. 61, 1956 (1992).

27. Y. C. Chang, L. L. Chang, and L. Esaki, "A New One-Dimensional Quantum Well Structure", Appl Phys. Lett. 47, 1324 (1985).

28. W. Wegscheider, L. N. Pfeiffer, M. M. Dignam, A Pinczuk, K. W. West, S. L. McCall, and R. Hull, "Lasing from Excitons in Quantum Wires", Phys. Rev. Lett. 71, 4071 (1993).

29. R. Dingle, "Confined Carrier Quantum States in Ultrathin Semiconductor Heterostructures", in Festkorperprobleme (Advances in Solid State Physics), Vol. 15, (NewYork, Pergamon, 1975). 
30. C. Kittel, "Introduction to Solid State Physics", 6th Ed. (New York: Wiley, 1986).

31. W. Kohn, and J. M. Luttinger, "Theory of Donor States in Silicon", Phys Rev. 98, 915 (1955).

32. D. A. Fraser, "The Physics of Semiconductor Physics", 4th Ed. (Oxford, UK: Clarendon Press, 1990).

33. L. I. Schiff, "Quantum Mechanics", 3rd Ed. (New York: McGraw-Hill, 1968).

34. R. L. Liboff, "Introductory Quantum Mechanics", (New York: Addison-Wesley, 1992).

35. N. W. Ashcroft and N. David Mermin, "Solid State Physics", (New York: Harcourt Brace Jovanovich, 1976).

36. G. H. Golub and C. F. Van Loan, "Matrix Computations", 2nd Ed. (Baltimore: John Hopkins University Press, 1989).

37. W. H. Press, B. P. Flannery, S. A. Teukolsky, and W. T. Vetterling, "Numerical Recipes in C", 2nd Ed. (Cambridge, UK: Cambridge University Press, 1992).

38. F. S. Acton, "Numerical Methods That Work", corrected Ed. (Washington: Mathematical Association of America, 1990).

39. W. H. Press, B. P. Flannery, S. A. Teukolsky, and W. T. Vetterling, "Numerical Recipes Example Book", 2nd Ed. (Cambridge, UK: Cambridge University Press, 1992).

40. M. Abramowitz and I. A. Stegun, "Handbook of Mathematical Functions", 9th Ed. (New York: Dover, 1970). 


\section{APPENDIX}

This program calculates the eigenvalues of Eq. (3-22) for the Q Dot discussed in Chapter 4 for both cases of an electron with and without an impurity.

\begin{tabular}{|c|c|c|}
\hline & Q_DOT.c & \\
\hline & $\begin{array}{l}\text { This is the main program. It is the most general program for the calculation of } \\
\text { eigenvalues of the real, nonsymmetric matrix SiH. The program finds the matrix }\end{array}$ & $*$ \\
\hline & elements of $S, T, V$, and $H$ by calling the subroutine agauss() which performs eight- & \\
\hline & and sixteen-point Gauss-Legendre integration. The inverse of matrix $S$ is calculated & \\
\hline & next, using LU decomposition and LU forward/backsubstitution through calls to & \\
\hline & the functions ludcmp() and lubksb() contained in th & * \\
\hline & After matrix_multiply $\mathrm{O}$ obtains the matrix $\mathrm{SiH}$, balanc $\mathrm{O}$ balances $\mathrm{SiH}$ to minimize & \\
\hline & rounding errors and then the subroutine elmhes $\mathrm{O}$ reduces $\mathrm{SiH}$ to upper Hessenberg & \\
\hline & $\begin{array}{l}\text { form. Finally, the subroutine hqr }(\text { performs a QR shift algorithm on matrix SiH } \\
\text { to calculate the eigenvalues. For the case of an impurity potential, the Energy is }\end{array}$ & 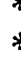 \\
\hline & output in units of the effective Rydberg, otherwise it is printed in terms of the unit & \\
\hline * & of energy of a three-dimensional spherical quantum well of radius $\mathrm{R}$. & \\
\hline & The subroutines ludcmp(), lubksb(), balanc(), elmhes(), and hqr() are from & \\
\hline & reference [37] (hereafter referred to as Numerical Recipes), and are listed below in & \\
\hline$*$ & the file qw_nr.c. The subroutines agauss(), inverse_matrix(), and matrix_multiply & \\
\hline & d below in file qw_other.c. Also listed below are other files needed by this & \\
\hline & & \\
\hline
\end{tabular}

\#include $<$ stdio.h $>$

\#include <math.h>

\#include "qw.h"

int i_el,j_el;

float LAMBDA, funcS(float r), funcV(float r), funcT(float $r$ );

int main(void)

\{

int $\mathrm{i}, \mathrm{j}, \mathrm{k}, \mathrm{noV}$;

float $\mathrm{S}, \mathrm{V}, \mathrm{T} 0, \mathrm{~T} 1, \mathrm{~T} 2$, coeff_t1, coeff_t 2 , binding_energy, radius;

float *ground_state_energy, ${ }^{*}$ energy_im, $* * \mathrm{~h},{ }^{* *} \mathrm{~s}, * * \mathrm{t},{ }^{*} * \mathrm{v},{ }^{*}{ }^{*} \mathrm{si}, * * \mathrm{sih}$;

float PREC $=0.000 \overline{2} ; \quad \digamma^{*}$ Precision required for final result of integration in agauss $(*)$

float FRAC $=0.0005 ; \quad \quad / *$ Minimum fraction of integration interval $* /$

for $(; ;)\{$

printf("InEnter $\mathrm{K}(0$ to quit): $\mathrm{n}$ ");

scanf("\%d",\&k);

/* Number of variational parameters */ 
printf(Radius $=\% 2.2 \mathrm{f}$ and Lambda $=\% 2,2 \mathrm{fn} "$, radius, LAMBDA);

if(noV) printf("No V, "); else printf("With V, ");

if(!noV) printf("Eb (Binding Energy) = E (Ground State Energy) - SQR(PI/R) $n "$ "); printf("Ground State Energy E in units of SQR(PI/R)\n");

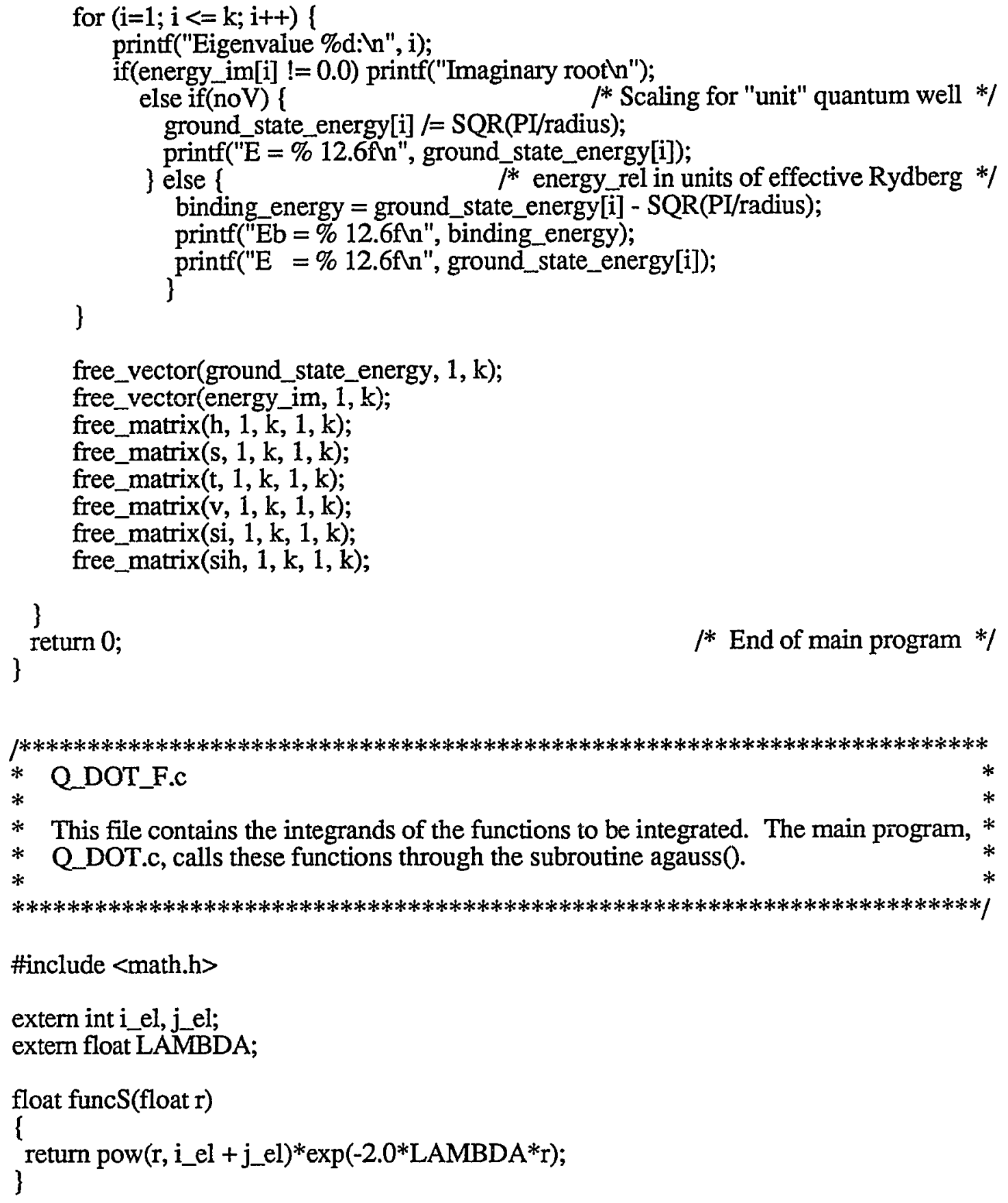



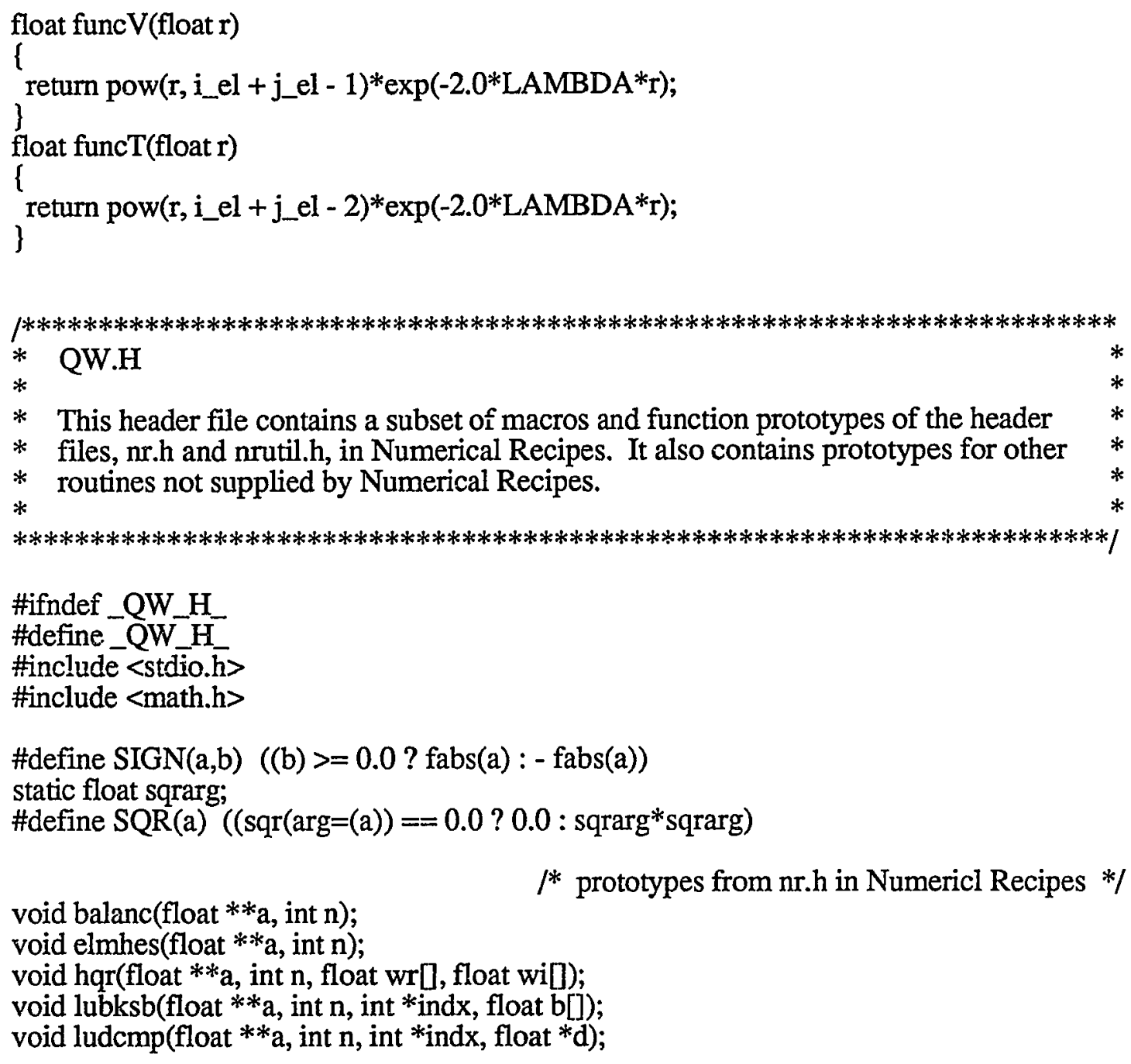

void nrerror(char error_text[]);

/* prototypes from nrutil.h in Numerical Recipes */ float *vector(long $\mathrm{nl}$, long $\mathrm{nh}$ ); int *ivector(long nl, long nh); float **matrix(long nrl, long $\mathrm{nrh}$, long ncl, long nch); void free_vector(float *v, long nl, long nh); void free_ivector(int *v, long $\mathrm{nl}$, long $\mathrm{nh}$ ); void free_matrix(float $* * \mathrm{~m}$, long $\mathrm{nrl}$, long $\mathrm{nrh}$, long ncl, long nch);

float agauss(float (*func)(float), float A, float B, float EPSLIN, float DELTA); * other prototypes *I void inverse_matrix (float $* * \mathrm{~m}$, float $* *$ mi, int $\mathrm{k}$ ); void matrix_multiply(float **new, float **old_a, float **old_b, int q); \#endif 


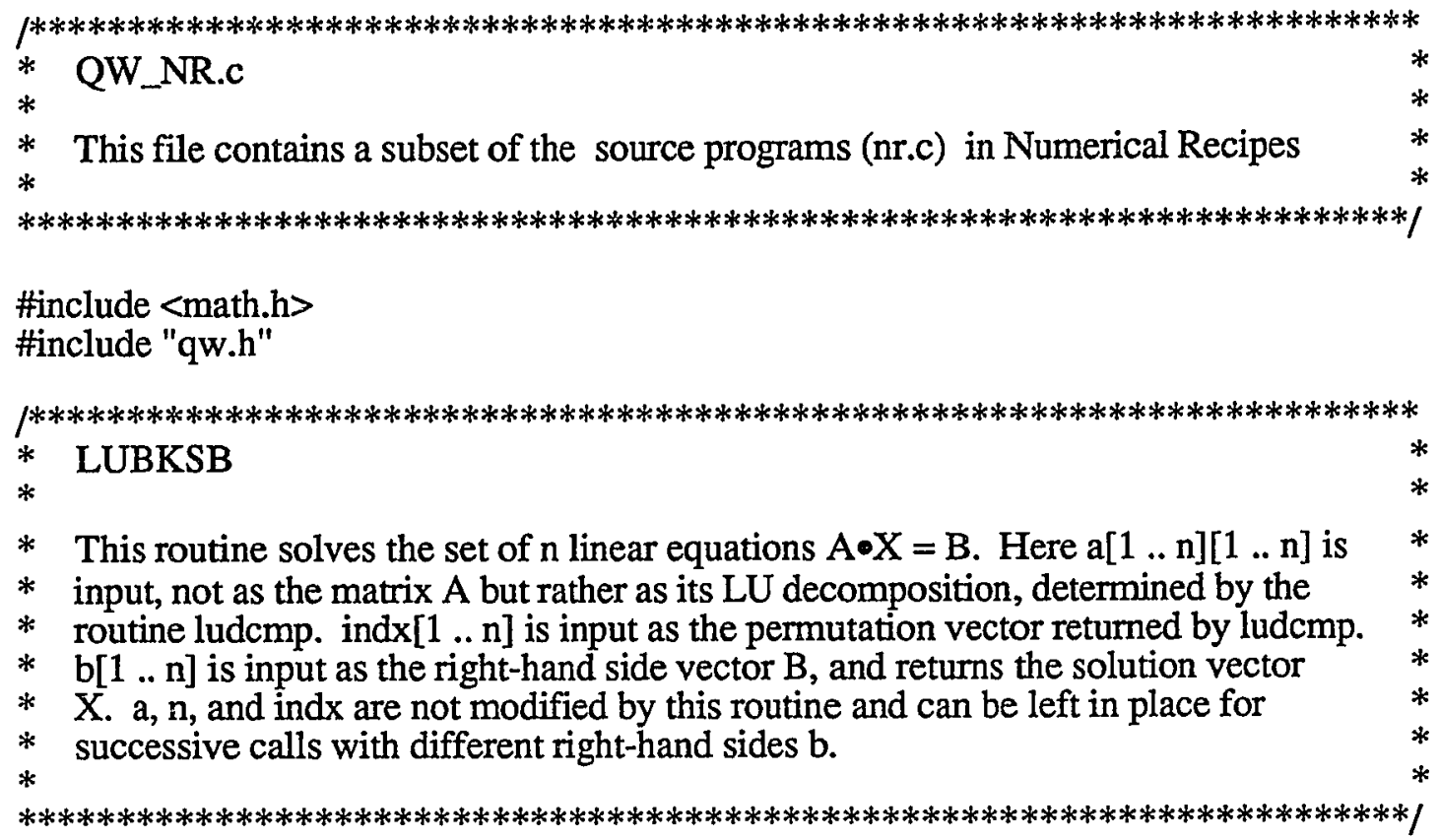

void lubksb(float $* * a$, int $\mathrm{n}$, int $*$ indx, float $\mathrm{b}[\mathrm{]})$;

\{

int $\mathrm{i}, \mathrm{ii}=0$, ip, $\mathrm{j}$;

float sum;

$1^{*}$ When ii is set to a positive value, it will become the index of the first nonvanishing element of $b$. We now do forward substitution, unscrambling permutations as we go

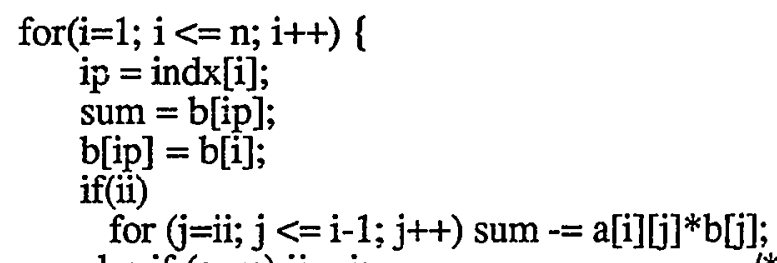

else if (sum) ii $=i$; \} $\mathrm{b}[\mathrm{i}]=$ sum; $\quad 1 *$ So from now on we will have to do the sums in the loop above */

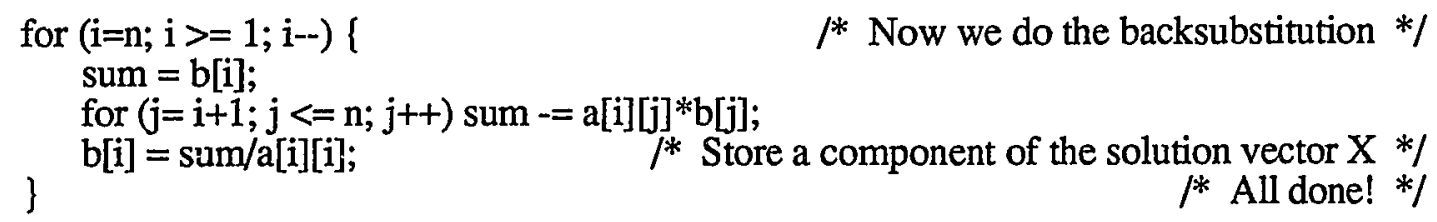




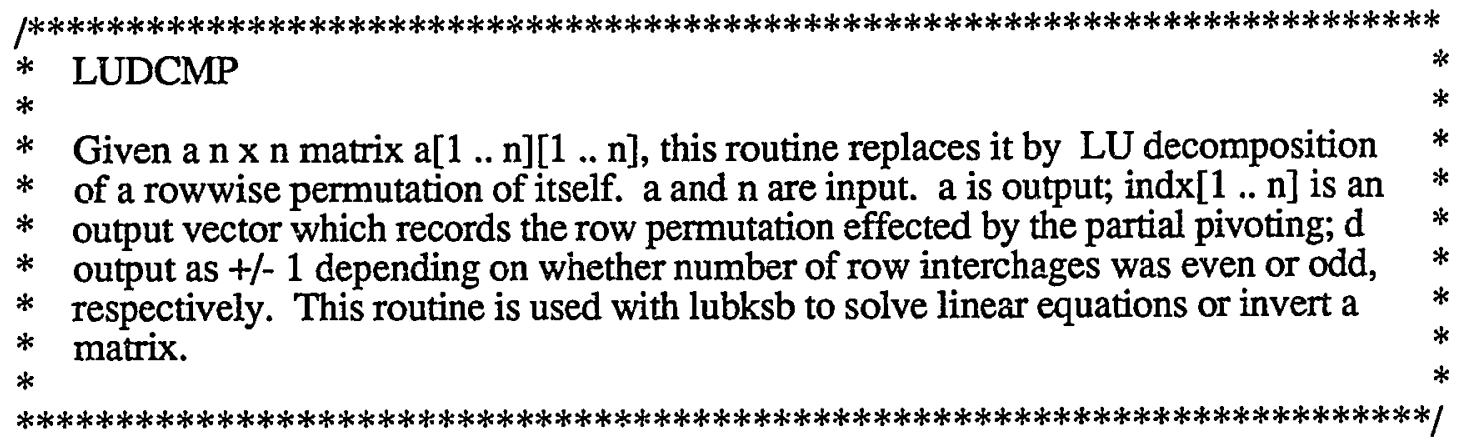

\#define TINY 1.0e-20

void ludcmp(float $* *_{\mathrm{a}}$, int $\mathrm{n}$, int $*_{\text {indx }}$, float $* \mathrm{~d}$ )

\{

int $\mathrm{i}$, imax, $\mathrm{j}, \mathrm{k}$;

float big, dum, sum, temp;

float *vv;

/* vv stores the implicit scaling of each row */

$\mathrm{vv}=\operatorname{vector}(1, \mathrm{n})$

$* \mathrm{~d}=1.0$

* No row interchanges yet $* I$

for $(\mathrm{i}=1 ; \mathrm{i}<=\mathrm{n} ; \mathrm{i}++)\{\quad / *$ Loop over rows to get the implicit scaling information $*\}$

big $=0.0$;

for $(j=1 ; j<=n ; j++)$ if $(($ temp $=$ fabs $(a[i][j]))>$ big $)$ big = temp;

if(big $==0.0$ ) nrerror("Singular matrix in routine ludcmp");

\}

$\mathrm{vv}[\mathrm{i}]=1.0 / \mathrm{big}$;

* No nonzero largest element $* /$

* Save the scaling */

for $(j=1 ; j<=n ; j++) \mid$

for $(\mathrm{i}=1 ; \mathrm{i}<\mathrm{j} ; \mathrm{i}++)\{$

sum $=\mathrm{a}[\mathrm{i}][\mathrm{j}]$;

for $(k=1 ; k<i ; k++)$ sum $-=a[i][k] * a[k][j] ;$

\} $\mathrm{a}[\mathrm{i}][\mathrm{j}]=$ sum;

big $=0.0$;

for $(i=j ; i<=n ; i++)\{$

sum $=\mathrm{a}[\mathrm{i}][\mathrm{j}]$;

for $(\mathrm{k}=1 ; \mathrm{k}<\mathrm{j} ; \mathrm{k}++)$ sum $=\mathrm{a}[\mathrm{i}][\mathrm{k}] * \mathrm{a}[\mathrm{k}][\mathrm{j}]$;

$\mathrm{a}[\mathrm{i}][\mathrm{j}]=$ sum;

* Is the figure of merit for the pivot bettern that the best so far? */

if $(($ dum $=v v[i] *$ fabs (sum) $)>=$ big $)\{$

big = dum;

\}

$\operatorname{imax}=\mathrm{i}$;

)

if(j ! = imax) \{

for $(k=1 ; k<=n ; k++)\{$

dum $=\mathrm{a}[\mathrm{imax}][\mathrm{k}]$

$\mathrm{a}[\mathrm{imax}][\mathrm{k}]=\mathrm{a}[\mathrm{j}][\mathrm{k}]$;

$/ *$ Do we need to interchange rows? */

/* Yes, do so ... */ 

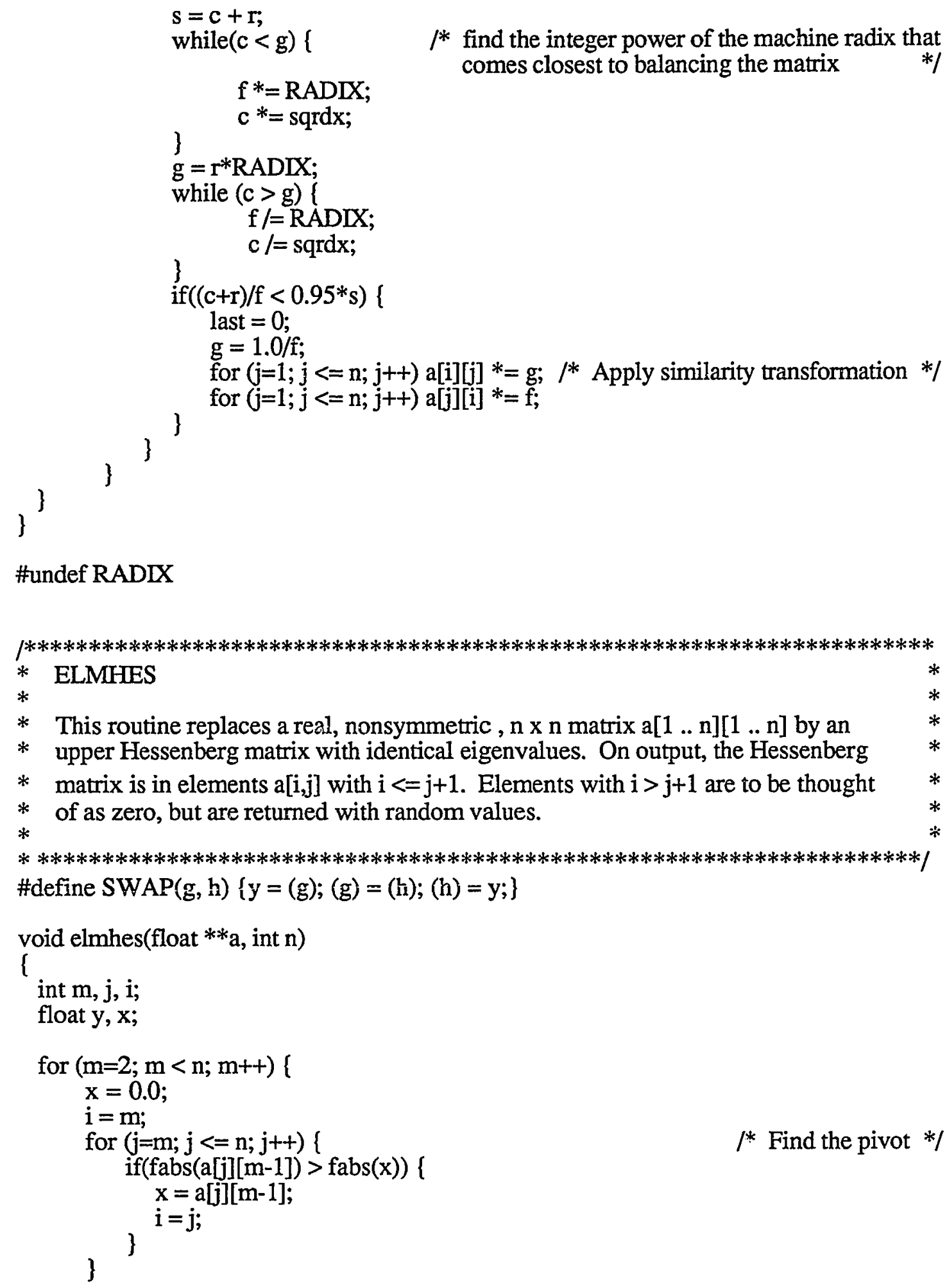


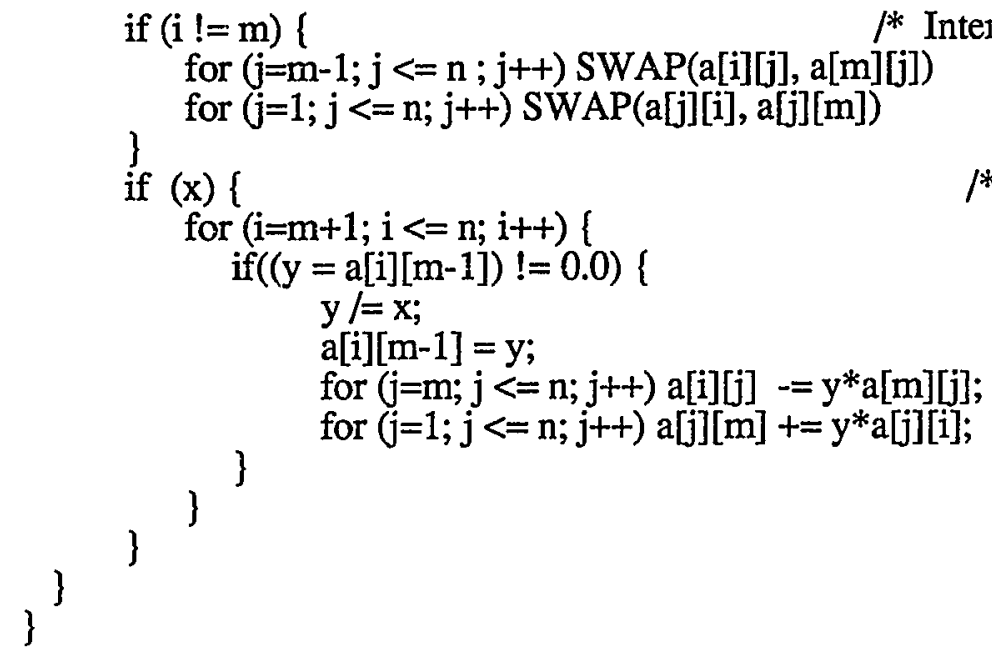

* Compute matrix norm for possible use in locating single small subdiagonal element */ for $(\mathrm{i}=2 ; \mathrm{i}<=\mathrm{n} ; \mathrm{i}++)$ 
$\mathrm{x}=\mathrm{a}[\mathrm{nn}][\mathrm{nn}] ;$

if $(\mathrm{I}==\mathrm{nn})$ ?

$\mathrm{wr}[\mathrm{nn}]=\mathrm{x}+\mathrm{t}$;

\}

wi[nn-- $]=0.0$;

$1 *$ One root found */

else 1

$\mathrm{y}=\mathrm{a}[\mathrm{nn}-1][\mathrm{nn}-1]$

$\mathrm{w}=\mathrm{a}[\mathrm{nn}][\mathrm{nn}-1] * \mathrm{a}[\mathrm{nn}-1][\mathrm{nn}]$

if $(1==(n n-1))$ \{

$p=0.5^{*}(y-x)$

$\mathrm{q}=\mathrm{p}^{*} \mathrm{p}+\mathrm{w}$

$\mathrm{z}=\operatorname{sqrt}(\mathrm{fabs}(\mathrm{q}))$;

$\mathrm{x}+\mathrm{t}$;

if $(q>=0.0)\{$

$\mathrm{z}=\mathrm{p}+\operatorname{SIGN}(\mathrm{z}, \mathrm{p})$

$w r[n n-1]=w r[n n]=x+z$

if $(\mathrm{z}) \mathrm{wr}[\mathrm{nn}]=\mathrm{x}-\mathrm{w} / \mathrm{z}$

\}

wi[nn-1] $=w i[n n]=0.0$;

/* Two roots found... */

else \{

$\operatorname{wr}[\mathrm{nn}-1]=\mathrm{wr}[\mathrm{nn}]=\mathrm{x}+\mathrm{p}$;

\} wi $[\mathrm{nn}-1]=-(\mathrm{wi}[\mathrm{nn}]=\mathrm{z})$;

$/^{*}$... a complex pair */

$\mathrm{nn}-=2$

\} else \{

/* No roots found. Continue iteration */

if(its $=30$ ) nrerror("Too many iterations in hqr");

if(its $==10 \|$ its $==20)$ (

$/ *$ Form exceptional shift */ $\mathrm{t}+=\mathrm{x}$;

for $(i=1 ; i<=n n ; i++)$ a[i] $[i]-=x$;

$\mathrm{s}=\mathrm{fabs}(\mathrm{a}[\mathrm{nn}][\mathrm{nn}-1])+\mathrm{fabs}(\mathrm{a}[\mathrm{nn}-1][\mathrm{nn}-2])$;

$\mathrm{y}=\mathrm{x}=0.75^{*} \mathrm{~s}$

\}

$\mathrm{w}=-0.4375^{*} \mathrm{~s}^{*} \mathrm{~s}$

++ its;

$I^{*}$ Form shift and then look for 2 consecutive small subdiagonal elements

for $(m=(n n-2) ; m>=I ; m--)\{$

$\mathrm{z}=\mathrm{a}[\mathrm{m}][\mathrm{m}]$

$\mathrm{r}=\mathrm{x}-\mathrm{z}$

$\mathrm{s}=\mathrm{y}-\mathrm{z}$

$\mathrm{p}=\left(\mathrm{r}^{*} \mathrm{~s}-\mathrm{w}\right) / \mathrm{a}[\mathrm{m}+1][\mathrm{m}]+\mathrm{a}[\mathrm{m}][\mathrm{m}+1]$;

$\mathrm{q}=\mathrm{a}[\mathrm{m}+1][\mathrm{m}+1]-\mathrm{z}-\mathrm{r}-\mathrm{s}$;

$\mathrm{r}=\mathrm{a}[\mathrm{m}+2][\mathrm{m}+1]$;

* Scale to prevent overflow or underflow */

$s=f a b s(p)+f a b s(q)+f a b s(r) ;$

$\mathrm{p} /=\mathrm{s}$;

$\mathrm{q} /=\mathrm{s}$;

$\mathrm{r} /=\mathrm{s}$;

if $(\mathrm{m}==1)$ break;

$\mathrm{u}=\mathrm{fabs}(\mathrm{a}[\mathrm{m}][\mathrm{m}-1]) *(\mathrm{fabs}(\mathrm{q})+\mathrm{fabs}(\mathrm{r}))$; 


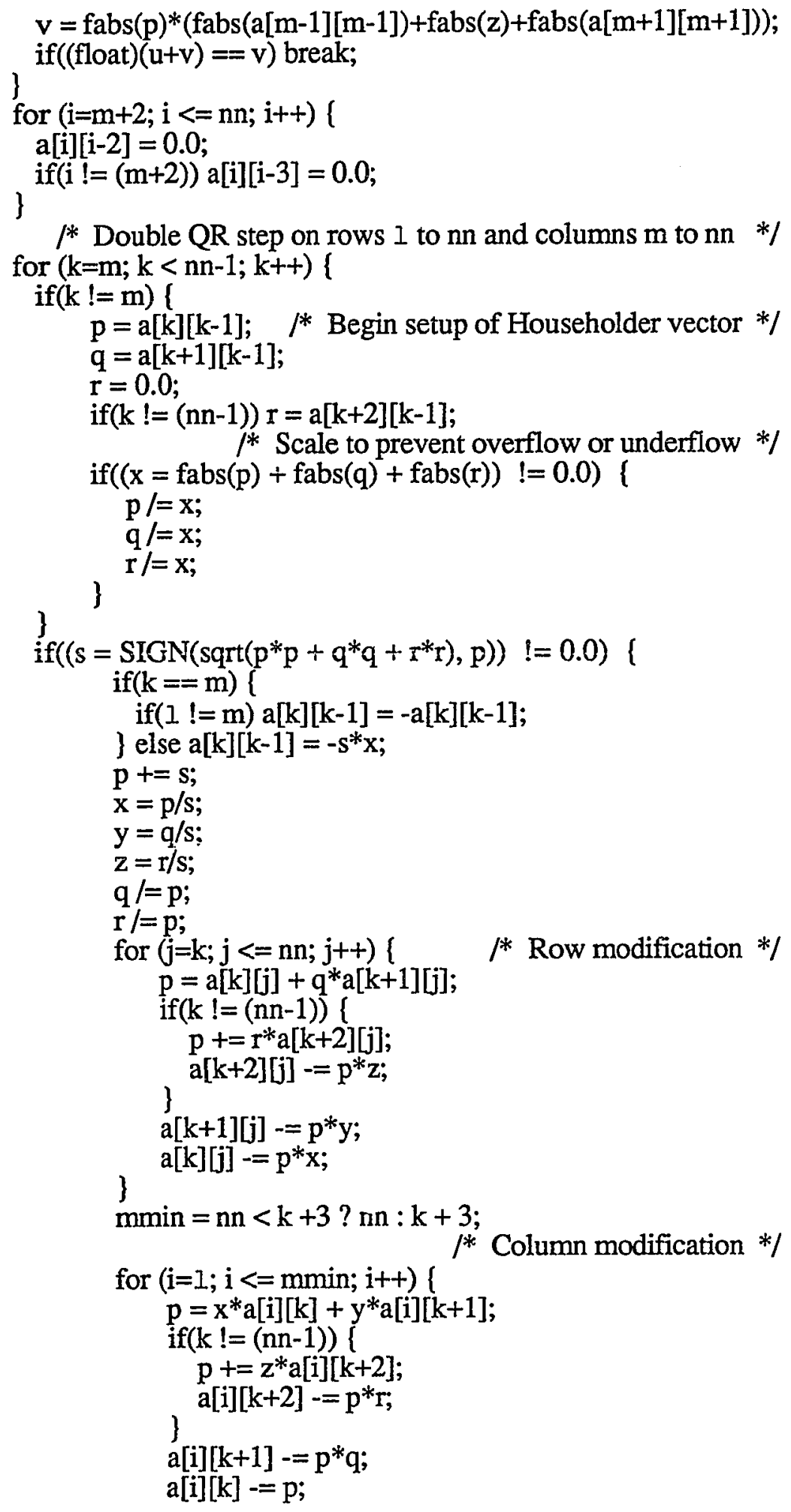




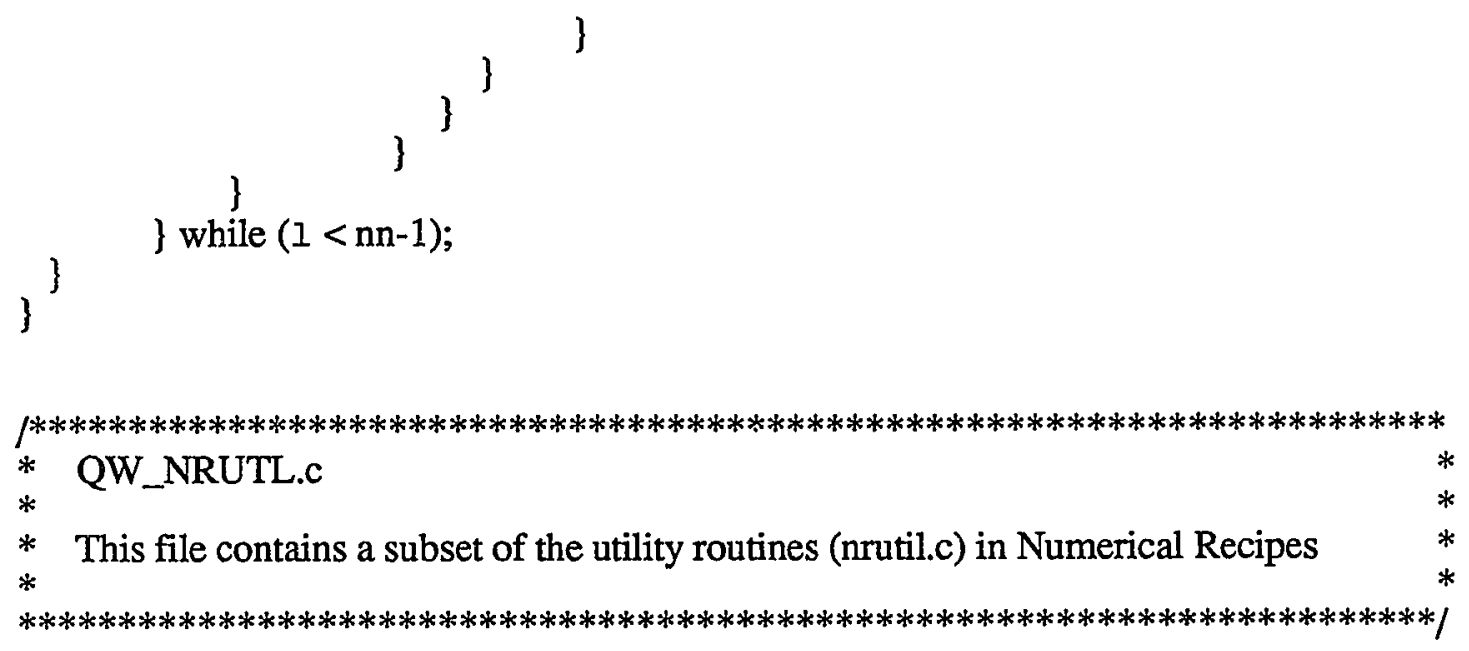

\#include $<$ stdio.h $>$

\#include <stddef.h $>$

\#include <stdlib.h>

\#define NR_END 1

\#define FREE_ARG char*

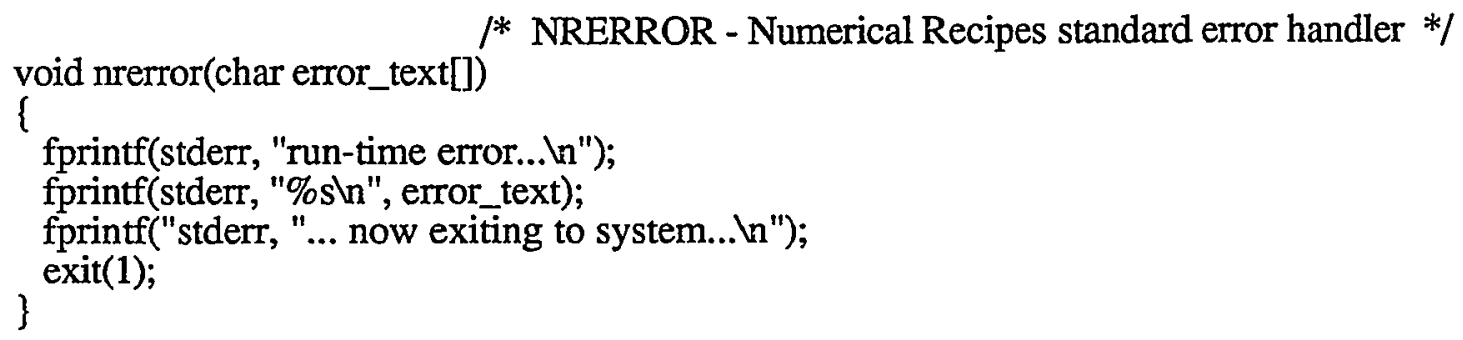

/* NRERROR - Numerical Recipes standard error handler */

\{

fprintf(stderr, "run-time error...tn"); fprintf(stderr, "\%sın", error_text); fprintf("stderr, "... now exiting to system... $n ")$;

\} exit(1);

float *vector(long $\mathrm{n} l$, long $\mathrm{nh}$ )

/* VECTOR - allocate a float vector with subscript range v[n] .. nh] */

\{

float *v;

$\mathrm{v}=($ float $*)$ malloc $\left((\right.$ size_t $)\left(\left(\mathrm{nh}-\mathrm{nl}+1+\mathrm{NR} \_\mathrm{END}\right) *\right.$ sizeof(float $\left.\left.)\right)\right)$;

if(!v) nrerror("allocation failure in vector()");

\} return $\mathrm{v}-\mathrm{nl}+\mathrm{NR}$ _END;

/* IVECTOR - allocate an int vector with subscript range v[nl .. nh] */ int $*_{\text {ivector(long nl, long } \mathrm{nh} \text { ) }}$

\{

int *v;

$\mathrm{v}=($ int $*) \operatorname{malloc}\left(\left(\right.\right.$ size $\left.\_\mathrm{t}\right)\left(\left(\mathrm{nh}-\mathrm{nl}+1+\mathrm{NR} \_\right.\right.$END $) *$ sizeof(int $\left.\left.)\right)\right)$;

if(!v) nrerror("allocation failure in ivector()");

\} return $\mathrm{v}-\mathrm{nl}+\mathrm{NR}$ END; 
${ }^{*}$ MATRIX - allocate a float matrix with subscript range m[nrl .. nrh][ncl .. nch] */ float **matrix(long nrl, long nrh, long ncl, long nch)

(

long $\mathrm{i}$, nrow $=\mathrm{nrh}-\mathrm{nrl}+1, \mathrm{ncol}=\mathrm{nch}-\mathrm{ncl}+1$

float **m;

$I^{*}$ allocate pointers to rows $* /$

$\mathrm{m}=($ float $* *)$ malloc $\left(\left(\right.\right.$ size $\left.\_t\right)(($ nrow + NR_END $) *$ sizeof $($ float $\left.*))\right)$;

if (!m) nrerror("allocation failure 1 in matrix ()$\left.^{\prime \prime}\right)$;

$\mathrm{m}+=$ NR_END;

$\mathrm{m}-=\mathrm{nrl}$;

$i^{*}$ allocate rows and set pointers to them $* /$

$\mathrm{m}[\mathrm{nrl}]=($ float $*)$ malloc $\left((\right.$ size_t $)\left(\left(\right.\right.$ nrow ${ }^{*}$ ncol $+\mathrm{NR} \_$END $) *$ sizeof(float $\left.\left.)\right)\right)$;

if (!m[nrl]) nrerror("allocation failure 2 in matrix ()$^{\prime \prime)}$;

$\mathrm{m}[\mathrm{nrl}]+=$ NR_END;

$\mathrm{m}[\mathrm{nrl}]-=\mathrm{ncl}$;

for $(i=n r l+1 ; i<=n r h ; i++) m[i]=m[i-1]+n c o l ;$

$/^{*}$ return pointer to array of pointers to rows $* /$

) return $\mathrm{m}$;

void free_vector(float $*$, long $\mathrm{nl}$, long $\mathrm{nh}$ )

\{

)

free $\left(\left(F R E E \_A R G\right)\left(v+n l-N R \_E N D\right)\right)$;

I* FREE_IVECTOR - free an int vector allocated with ivector $0 *$ /

void free_ivector(int $* \mathrm{v}$, long $\mathrm{nl}$, long $\mathrm{nh}$ )

\{

\}

free((FREE_ARG) $(\mathrm{v}+\mathrm{nl}$ - NR_END));

/* FREE_MATRIX - free a float matrix allocated by matrix() */

void free_matrix(float $* * \mathrm{~m}$, long $\mathrm{nrl}$, long $\mathrm{nrh}$, long ncl, long $\mathrm{nch}$ )

l

free ((FREE_ARG) (m[nrl] + ncl - NR_END));

\}

free ((FREE_ARG) $(\mathrm{m}+\mathrm{nrl}-\mathrm{NR}$ _END));

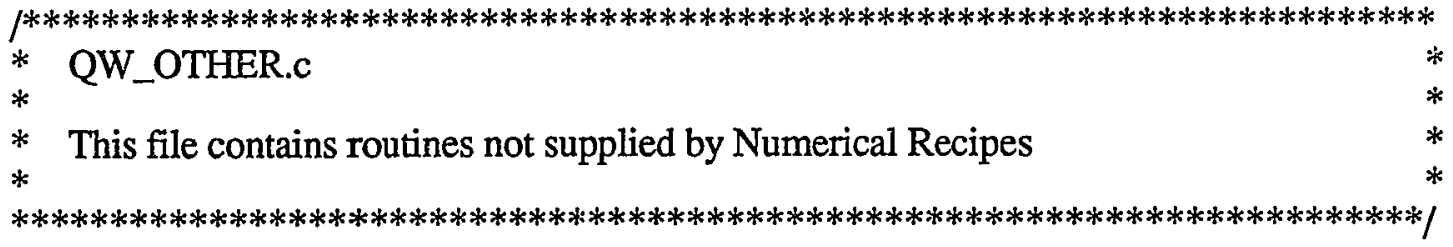

\#include <stdio.h $>$

\#include <math.h>

\#include "qw.h" 
\#define SUM(W, X) $\left((W) *\left((*\right.\right.$ func $\left.\left.)\left((\mathrm{C} 1)+\left((\mathrm{C} 2)^{*}(\mathrm{X})\right)\right)\right)\right)$

float agauss(float (*func)(float), float $A$, float $B$, float EPSLIN, float DELTA) \{ int $\mathrm{N}$;

float gauss, AA, BB, Y, S8, S16, C1, C2, C3;

gauss $=0$

$\mathrm{AA}=\mathrm{A}$;

part5: $\mathrm{Y}=\mathrm{B}-\mathrm{AA}$;

if((DELTA - fabs(Y)) > 0) goto part1;

part2: $\mathrm{BB}=\mathrm{AA}+\mathrm{Y}$;

$\mathrm{C} 1=0.5^{*}(\mathrm{AA}+\mathrm{BB})$;

$\mathrm{C} 2=\mathrm{C} 1-\mathrm{AA}$;

$\mathrm{C} 3=\mathrm{C} 2$;

$\mathrm{N}=1$

$\mathrm{S} 8=0$;

$S 16=0$;

part8: $\mathbf{S} 8=\mathbf{S} 8+$

SUM( 0.101228536290376, 0.960289856497536) r SUM( 0.222381034453374, 0.796666477413627) + SUM( 0.313706645877887, 0.525532409916329) + SUM( 0.362683783378362, 0.183434642495650);

$\mathrm{S} 16=\mathrm{S} 16+$

SUM( 0.027152459411754, 0.989400934991650)+ SUM( 0.062253523938648, 0.944575023073233) + SUM( 0.095158511682493, 0.865631202387832) + SUM( 0.124628971255534, 0.755404408355003) + SUM( 0.149595988816577, 0.617876244402644) + SUM( 0.169156519395003, 0.458016777657227) + SUM( 0.182603415044924, 0.281603550779259) + SUM( 0.189450610455069, 0.095012509837637); 


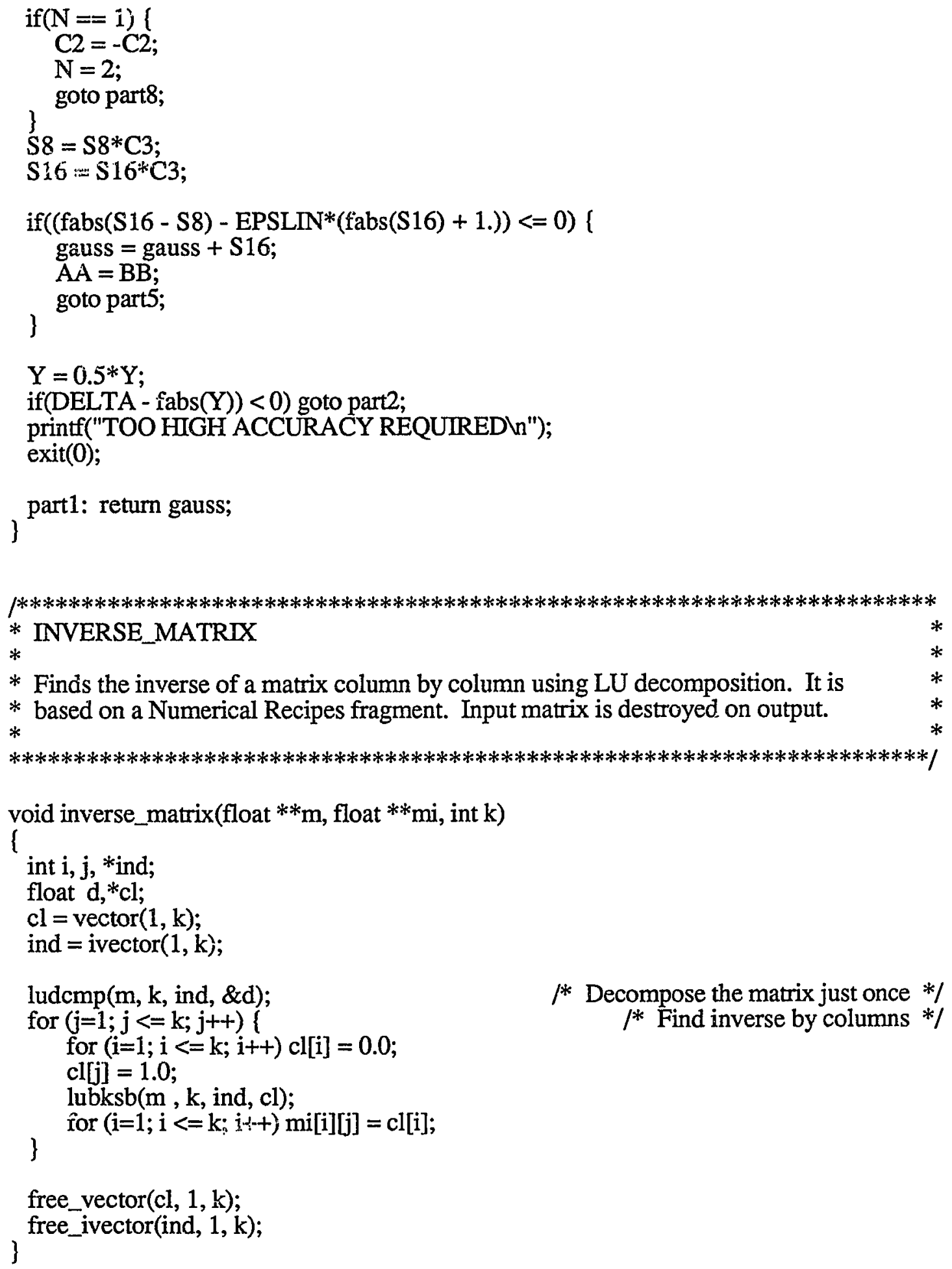

* Decompose the matrix just once */ $l^{*}$ Find inverse by columns $* /$ 
void matrix_multiply(float **new, float **old_a, float **old_b, int q)

\{ int $i, j, k$;

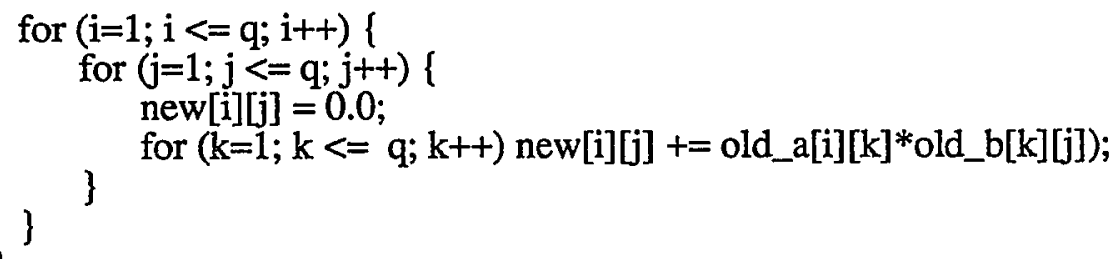

